\title{
Structure and mechanism of the mammalian fructose transporter GLUT5.
}

\section{AUTHOR(S):}

Nomura, Norimichi; Verdon, Grégory; Kang, Hae Joo; Shimamura, Tatsuro; Nomura, Yayoi; Sonoda, Yo; Hussien, Saba Abdul; ... Kasahara, Michihiro; Iwata, So; Drew, David

\section{CITATION:}

Nomura, Norimichi ...[et al]. Structure and mechanism of the mammalian fructose transporter GLUT5.. Nature 2015, 526(7573): 397401

\section{ISSUE DATE:}

2015-09-30

\section{URL:}

http://hdl.handle.net/2433/199936

\section{RIGHT:}

(c) 2015 Macmillan Publishers Limited.; The full-text file will be made open to the public on 30 march 2016 in accordance with publisher's 'Terms and Conditions for Self-Archiving'.; この論文は出版社版でありません。引用の際には出版社版をご確認ご利用ください。;

This is not the published version. Please cite only the published version. 


\section{Structure and mechanism of the mammalian fructose transporter GLUT5}

Norimichi Nomura ${ }^{1,2,3 * \# \text {, Grégory } \operatorname{Verdon}^{4,5,6 *} \text {, Hae Joo Kang }}{ }^{4,5,6 *}$, Tatsuro Shimamura $^{1,2,3}$, Yayoi Nomura ${ }^{1,2,3}$, Yo Sonoda ${ }^{4}$, Saba Abdul Hussien ${ }^{7}$, Aziz Abdul Qureshi $^{7}$, Mathieu Coincon ${ }^{7}$, Yumi Sato ${ }^{1,3}$, Hitomi Abe ${ }^{1}$, Yoshiko Nakada-Nakura ${ }^{1,3}$, Tomoya Hino ${ }^{1,2}$, Takatoshi Arakawa ${ }^{1,2}$, Osamu Kusano-Arai ${ }^{8}$, Hiroko Iwanari ${ }^{8}$, Takeshi Murata ${ }^{1,2,3,9}$, Takuya Kobayashi ${ }^{1,2,3}$, Takao Hamakubo ${ }^{8}$, Michihiro Kasahara $^{10}$, So Iwata ${ }^{1,2,3,4,5,6,9}$ \#, David Drew ${ }^{4,7} \#$

${ }^{1}$ Department of Cell Biology, Graduate School of Medicine, Kyoto University, Konoe-cho, Yoshida, Sakyo-ku, Kyoto 606-8501, Japan. ${ }^{2}$ Japan Science and Technology Agency, ERATO, Iwata Human Receptor Crystallography Project, Yoshida-Konoe-cho, Sakyo-ku, Kyoto 606-8501, Japan. ${ }^{3} J a p a n$ Science and Technology Agency, Research Acceleration Program, Membrane Protein Crystallography Project, Yoshida-Konoe-cho, Sakyo-ku, Kyoto 606-8501, Japan. ${ }^{4}$ Division of Molecular Biosciences, Imperial College London, London, SW7 2AZ, U.K. ${ }^{5}$ Membrane Protein Laboratory, Diamond Light Source, Harwell Science and Innovation Campus, Didcot, Chilton, Oxfordshire, OX11 0DE, U.K. ${ }^{6}$ Research Complex at Harwell Rutherford, Appleton Laboratory, Harwell, Oxford, Didcot, Oxfordshire, OX11 0FA, U.K. ${ }^{7}$ Centre for Biomembrane Research, Department of Biochemistry and Biophysics, Stockholm University, SE-106 91 Stockholm, Sweden. ${ }^{8}$ Department of Quantitative Biology and Medicine, Research Center for Advanced Science and Technology, University of Tokyo, 4-6-1 Komaba, Meguro-ku, Tokyo 153-8904, Japan. ${ }^{9}$ Systems and Structural Biology Center, RIKEN, 1-7-22 Suehirocho, Tsurumi-ku, Yokohama 230-0045, Japan. ${ }^{10}$ Laboratory of Biophysics, School of Medicine, Teikyo University, Hachioji, Tokyo 192-0395, Japan.

*These authors contributed equally

\#Correspondence:

nnomura@mfour.med.kyoto-u.ac.jp

d.drew@dbb.su.se

s.iwata@mfour.med.kyoto-u.ac.jp 


\section{Abstract}

The altered activity of the fructose transporter GLUT5, an isoform of the facilitated-diffusion glucose transporter family, has been linked to disorders such as type 2 diabetes and obesity. GLUT5 is also overexpressed in certain tumor cells and inhibitors are potential drugs for these conditions. Here, we describe the crystal structure of GLUT5 from Rattus norvegicus and Bos taurus in open outward- and open inward-facing conformations, respectively. GLUT5 has a major facilitator superfamily fold like other homologous monosaccharide transporters. Based on a comparison of the inward-facing structures of GLUT5 and human GLUT1, a ubiquitous glucose transporter, we show that a single point mutation is enough to switch the substrate binding preference of GLUT5 from fructose to glucose. A comparison of the substrate-free structures of GLUT5 with occluded substrate-bound structures of XylE suggests that, besides global rocker-switch like re-orientation of the bundles, local asymmetric rearrangements of C-terminal bundle helices TMs 7 and 10 underlie a "gated-pore" transport mechanism in such monosaccharide transporters.

\section{Introduction}

GLUT transporters belong to the solute carrier 2 family (SLC2) and, so far, fourteen different isoforms GLUT1-14 have been identified ${ }^{1,2}$. Except for GLUT13, GLUT transporters are uniporters, which facilitate the diffusion of monosaccharides like glucose and fructose across the cell membrane in a concentration-dependent manner ${ }^{1,2}$. Each GLUT transporter shows a distinct pattern of tissue distribution, gene regulation, substrate preference and kinetic properties $^{1,2}$. For example, GLUT1 is distributed in a wide range of tissues, including the blood-brain barrier and is essential for glucose transport into the brain ${ }^{3}$, whereas GLUT4 is mostly localized to skeletal muscles and adipose tissue, and is the major insulin-stimulated glucose transporter ${ }^{1,4}$. GLUT5 is the only member specific to fructose ${ }^{5,6}$ and together with GLUT2, which transports fructose in addition to glucose, they make up the major fructose transporters in the body ${ }^{7,8}$. GLUT5 is primarily expressed in the small intestine, but lower levels 
are also expressed in brain, adipose tissue, kidney, testes and skeletal muscle $5,9,10$. GLUT activity is also associated with various diseases ${ }^{1,11}$. For instance, increased GLUT5 expression has been linked to several metabolic disorders ${ }^{12,13}$, and several types of cancers like breast cancer ${ }^{14}$, because of the higher energy demand of cancer cells stimulating sugar uptake, the so-called Warburg effect ${ }^{15}$. GLUT transporters belong to the larger major facilitator superfamily (MFS), whose members have a fold consisting of two symmetrical six transmembrane helix (TM) bundles ${ }^{16,17}$. Within the MFS they belong to a subfamily of sugar porter transporters ${ }^{18,19}$, whose members are found in all domains of life and are important targets for industrial and biomedical applications ${ }^{20}$. Recently, an open inward-facing structure of human GLUT1 was reported with a bound sugar from a detergent head-group in the substrate-binding site, and compared to previous structures of the related Escherichia coli D-xylose: $\mathrm{H}^{+}$symporter XylE in the outward- and inward-occluded conformations, suggesting a "rocker-switch" type transport mechanism ${ }^{21-23}$. However, as little is known about the molecular basis of substrate binding and release in GLUT transporters, their alternating-access mechanism is yet to be fully understood.

\section{Open outward and inward GLUT5 structures}

Rat and bovine GLUT5 (rGLUT5 and bGLUT5) that share 81\% sequence identity to human GLUT5 were selected and optimised for structural studies using fluorescence-based screening methods (Methods). rGLUT5 was crystallized in complex with an Fv antibody fragment (rGLUT5-Fv, Methods). The rGLUT5-Fv and bGLUT5 structures were solved by molecular replacement (MR) and refined against data extending up to $3.3 \AA$ and 3.2/4.0 $\AA$ (anisotropic data), respectively (Extended Data Table 1 and 2, Extended Data Fig 1, and Methods). The GLUT5 structure shows the typical MFS fold, plus five additional helices on the intracellular side, one at the C-terminus (ICH5) and the other four, ICH1-4, located between the N- and C-terminal TM bundles (Fig. 1). bGLUT5 crystallized in an open inward-facing conformation (Fig. 1) and although human GLUT1 (hGLUT1) and bGLUT5 share only $43 \%$ sequence identity, their inward-facing structures superimpose well, with an r.m.s.d. of $1.12 \AA$ for 364 pairs of $\mathrm{C} \alpha$ atoms (Methods and Extended Data Fig. 2a). The rGLUT5-Fv structure shows an open 
outward-facing conformation, which is a state that has not been observed previously in any of the related sugar porter structures ${ }^{22-25}$ (Fig. 1). The open outward-facing conformation is possibly stabilized by the Fv fragment, which binds to the ICHs (Extended Data Fig. 3).

\section{Central fructose-binding site of GLUT5}

The GLUT5 substrate-binding site is closely related to those of hGLUT1 and E. coli XylE21,22 (Fig. 2a and Extended Data Fig. 2b). Many of the residues lining the central cavity are conserved between GLUT5 and hGLUT1, and include Ile169, Ile173, Gln166, Gln287, Gln288, Asn324 and Trp419 (Fig. 2a and Extended Data Fig. 4). In GLUT5, Trp419 is the only tryptophan positioned in the substratebinding site (Fig. 2a and Extended Data Fig. 5a), and it is essential for transport 26 . Consistent with rGLUT5 transport activity (Extended Data Fig. 6a), strong quenching of tryptophan fluorescence could be observed with the addition of Dfructose, but not with the addition of L-fructose or known GLUT1 substrates like D-glucose, D-galactose or D-mannose (Extended Data Fig. 5). Using this assay, the affinity of rGLUT5 for D-fructose was measured to have a $K_{\mathrm{d}}$ around 6 - $9 \mathrm{mM}$ (Fig. 2b and Extended Data Fig. 6b), which is similar to that reported for human GLUT55,8. Throughout this study tryptophan fluorescence quenching of rGLUT5 was henceforth used to assess substrate binding.

Gln166 is the only conserved residue in the substrate-binding site that is positioned differently between the hGLUT1 and GLUT5 structures (Fig. 2a). In hGLUT1 the equivalent glutamine (Gln161) points away, rather than towards, the central cavity as it has likely re-orientated to accommodate the acyl chain of the bound detergent in the sugar-binding site (Fig. 2a). In XylE the equivalent glutamine (Gln168) is orientated similarly as in GLUT5 (Extended Data Fig. 2b). Other residues that line the binding site, but are not conserved between GLUT5 and hGLUT1 are Tyr31 (Phe26 in hGLUT1), His386 (Phe379), Ala395 (Trp388), His418 (Asn411) and Ser391 (Gly384) (Fig. 2a and Extended Data Fig. 4). Single alanine mutants of each of these residues show strongly reduced D-fructose binding (Fig. 2c). The alanine mutants that show the weakest D-fructose binding are Tyr31 (TM1), Gln287 (TM7), His386 (TM10) and His418 (TM11). Except for 
Tyr31, these residues belong to the C-terminal TM bundle, indicating an asymmetrical binding mode of the sugar between the $\mathrm{N}$ - and C-terminal TM bundles in GLUT5, as seen in the sugar-bound hGLUT1 and XylE structures ${ }^{21,22}$. The substrate-binding cavity is deeper in GLUT5, because the tryptophan residue found at the bottom of the cavity in hGLUT1 (Trp388) and XylE (Trp392) is replaced with alanine (Ala395) in GLUT5 (Fig. 2a and Extended Data Fig. 6c). Trp388 in hGLUT1 is critical for inhibition by cytochalasin $\mathrm{B}^{26}$, whereas GLUT5 is insensitive to this inhibitor ${ }^{5}$.

GLUT7 is the closest isoform to GLUT5 and transports both D-fructose and Dglucose ${ }^{27}$. Among the substrate-binding site residues of GLUT5 almost all are identical to those in GLUT7 (Extended Data Fig. 4). The most notable difference is that Gln166 in GLUT5 is replaced in GLUT7 with glutamate (Extended Data Fig. 4). In rGLUT5, the substitution of Gln166 (TM5) with glutamate clearly weakens D-fructose binding (Fig. 2c and 2d). Furthermore, the Q166E mutant now shows robust binding to D-glucose with an apparent affinity $\left(K_{\mathrm{d}}\right)$ of $\sim 4 \mathrm{mM}$ (Fig. $2 \mathrm{~d}$ and Fig. 2e). The introduced carboxylate is likely to play a similar role to Glu380 (TM10) in hGLUT1 (Fig. 2a), which is essential for D-glucose transport28.

\section{Salt-bridges stabilize the outward conformation}

A common feature observed in recent structures of MFS transporters are interTM bundle salt-bridges that break and form near the central cavity during transport $16,29,30$. In $\mathrm{H}^{+}$-coupled transporters, it is thought that the protonation state of these salt-bridges is coupled to substrate-binding and further to structural transitions ${ }^{16,29,30}$. In GLUT5, no salt-bridges are observed near the central cavity in either conformation. Instead, inter-TM bundle salt-bridges are observed only in the outward-facing conformation and far from the central cavity, linking the cytoplasmic ends of TMs 3, 4, 5 in the N-terminal TM bundle to those of TMs 9, 10 and 11 in the C-terminal TM bundle (Fig. 3a). Specifically, Glu151 (TM4) forms a salt-bridge to both Arg97 (TM3) and Arg407 (TM11), and similarly Glu400 (TM10) forms a salt-bridge to both Arg158 (TM5) and Arg340 (TM9). Due to their strict conservation these residues make up the welldescribed sugar porter signatures ${ }^{18,19}$ and are related by a pseudo two-fold 
symmetry axis running through the center of the transporter and perpendicular to the membrane plane (Fig. 1a and Fig. 3b and Extended Data Fig. 4). In the inward-facing conformation the inter-TM bundle salt-bridge pairs Glu400Arg158 and Glu151-Arg407 are separated by some $\sim 7$ and $13 \AA$, respectively (Fig. 3a). In the inward-facing conformation, no inter-TM bundle salt-bridges are formed on the extracellular side, indicating that in the absence of substrate GLUT proteins may favor the outward-facing conformation. Consistently, the substitution of Glu400, Arg407 and Glu336 equivalent residues in GLUT4 to neutral amino acids arrests the transporter in an inward-facing conformation ${ }^{31}$. Moreover, in the outward-facing conformation Glu336 (TM8) in the C-terminal TM bundle forms a salt-bridge to Arg340, which is connected to the inter-TM bundle Glu400-Arg158 salt-bridge (Fig. 3a). The equivalent glutamate to Glu336 in hGLUT1 (Glu329) was substituted to glutamine to stabilize the inward-facing hGLUT1 structure ${ }^{21}$. The salt-bridges formed between inter-TM bundles seem to be coupled also to substrate-binding in GLUT5, because substituting Glu151 and Glu400 with alanine shows significantly reduced D-fructose binding (Fig. 3c).

The ICH domain is below this cytoplasmic salt-bridge network, and positioned similarly, with respect to the N- and C-terminal TM bundles, in both outwardopen GLUT5 and outward-occluded XylE structures ${ }^{22}$ (Extended Data Fig. 7a). ICH1, ICH2 and ICH3 are linked together by salt-bridges, as previously shown for XylE and GLUT121,22. In GLUT5, ICH5 lacks charged residues that could interact with ICH1-3 (Extended Data Fig. 7b), as it does in the outward-occluded XylE structure $^{22}$. Rather, the N-terminal helices ICH1-3 interact with the C-terminal bundle via a salt-bridge formed between Glu252 in ICH3 and Arg407 in TM11; thus linking the ICH domain to a TM involved in the inter-bundle salt-bridge network. In inward-facing GLUT5, these interactions are broken (Extended Data Fig. 7c) and, as observed in the inward-facing hGLUT1 and XylE structures ${ }^{21,23,24}$, ICH5 could not be built (Extended Data Fig. 2a). Therefore, the role of the ICH domain might be to provide additional stabilization of the outward-facing conformation, as suggested previously ${ }^{21}$. 


\section{TMs 7 and 10 form substrate-induced gates}

In GLUT5, the N- and C-terminal TM bundles undergo a small rotation of $\sim 15^{\circ}$ between the open outward- and inward-facing conformations (Fig. 1). As observed in other MFS transporter structures ${ }^{16}$, cavity-closing contacts in GLUT5 form mostly between TMs 1 and 7 on the outside, and between TMs 4 and 10 on the inside (Fig. 1c and Fig. 4a). Among these helices, however, TMs 7 and 10 in the C-terminal TM bundle seem to play the most prominent roles in occluding the substrate-binding site from the outside and inside, respectively (Fig. 4b). Comparisons of the substrate-free open GLUT5 structures with the substrateoccluded XylE structures 22,23 highlight the central role of TM7 and TM10 in gating (Extended Data Fig. 8). Inverted-symmetry related TMs 7 and 10 make up highly conserved sugar transporter signatures ${ }^{2}$, and they also form a large fraction of the substrate-binding site in hGLUT121 and XylE21,22, in agreement with previous functional data $28,32,33$. Between the outward-open GLUT5 conformation and outward-occluded XylE conformation ${ }^{22}$, the extracellular half of TM7 shows the largest shift towards the substrate-binding site (Extended Data Fig. 8a). Similarly, from the inward-occluded XylE conformation ${ }^{23}$ to the inward-open GLUT5 conformation, the intracellular half of TM10 shows the largest shift away from the substrate-binding site (Extended Data Fig. 8b and c); this TM10 movement has also been described previously for XylE 23,24. In GLUT5, the observed conformational changes in TMs 7 and 10 occur at hinge points that contain glycine residues (Fig. 4b and 4c and Extended Data Fig. 4). Tyr382 in TM10 interacts tightly with TM7 residues Ileu295 and Val292 in the outward-facing state and these interactions do not take place in the inward-facing conformation (Fig. 4c). The interactions between TMs 7 and 10 seem important to coordinate their conformational changes during transport, because mutation of the Ile295 equivalent residue in hGLUT5 to valine or alanine abolishes D-fructose transport ${ }^{34}$. Consistently, similar interface-perturbing mutations of Ile295 significantly reduce D-fructose binding in rGLUT5 (Extended Data Fig. 6d). Further, in hGLUT1 the equivalent residue to Val292 (Ile287) was substituted to every other amino acid, and each mutant shows markedly different D-glucose affinities and transport kinetics ${ }^{35}$. Therefore, it suggests that the interactions 
between TM7 and TM10 are important and tuned with respect to substrate affinity and transport kinetics.

\section{Conclusions}

Symmetrical substrate binding and rigid-body movements of the $\mathrm{N}$ - and Cterminal TM bundles around a centrally located substrate-binding site form the structural basis of the "rocker-switch" mechanism in MFS transporters ${ }^{16,36}$. However, here we have described asymmetrical rearrangements in the MFS subfamily of sugar porters, consistent with the asymmetrical binding mode of the sugar reported previously in XylE and hGLUT1 structures ${ }^{21,22}$. Therefore, we conclude that transport in GLUT5 is not only controlled by the rocker-switch type movement ${ }^{21}$ of the $\mathrm{N}$ - and $\mathrm{C}$-terminal TM bundles, but also by a gated-pore mechanism, in the form of local, gating movements by TMs 7 and 10 in the Cterminal TM bundle that are coupled to substrate binding and release (Fig. 5 and Supplementary Videos 1 - 3). TM10 is also part of the inter-TM bundle saltbridge network, indicating how local gating and global rocker-switch type movements are coupled. Importantly, a deeper understanding of GLUT5 structure and transport mechanism, as described here, should facilitate the structure-based design of novel inhibitors with therapeutic potential.

\section{METHODS}

Rattus norvegicus GLUT5 cloned full-length sequence (UniProt accession number: P43427); N50Y deglycosylation mutation is underlined and additional C-terminal residues retained after TEV cleavage are shown in italic (see next section for cloning details).

MEKEDQEKTGKLTLVLALATFLAAFGSSFQYGYNVAAVNSPSEFMQQFYYYDTYYDRNK ENIESFTLTLLWSLTVSMFPFGGFIGSLMVGFLVNNLGRKGALLFNNIFSILPAILMGCSK IAKSFEIIIASRLLVGICAGISSNVVPMYLGELAPKNLRGALGVVPQLFITVGILVAQLFGLR SVLASEEGWPILLGLTGVPAGLQLLLLPFFPESPRYLLIQKKNESAAEKALQTLRGWKD VDMEMEEIRKEDEAEKAAGFISVWKLFRMQSLRWQLISTIVLMTGQQLSGVNAIYYYA DQIYLSAGVKSNDVQYVTAGTGAVNVFMTMVTVFVVELWGRRNLLLIGFSTCLTACIVL TVALALQNTISWMPYVSIVCVIVYVIGHAVGPSPIPALFITEIFLQSSRPSAYMIGGSVHW 
LSNFIVGLIFPFIQVGLGPYSFIIFAIICLLTSIYIFMVVPETKGRTFVEINQIFAKKNKVSDV YPEKEEKELNDLPPATREQENLYFQ

Bos taurus GLUT5 cloned sequence containing 1 to 473 out of 501 residues (UniProt accession number: P58353); N51A deglycosylation mutation is underlined and additional C-terminal residues retained after TEV cleavage are shown in italic (see next section for cloning details).

MEPQDPVKREGRLTPVIVLATLIAAFGSSFQYGYNVAAINSPSEFMKDFYAYYTYYDRVG EYMNEFYLTLLWSVTVSMFPFGGFLGSLMVGPLVNNLGRKGTLLFNNIFSIVPALLMGF SELAKSFEMIIVARVLVGICAGLSSNVVPMYLGELAPKNWRGALGVVPQLFITIGILVAQI FGLRSLLANEEGWPILLGLTGIPAVLQLLFLPFFPESPRYLLIQKKDEAAAKSALRRLRG WHDVDAEIEEILEEDRAEKAVGFISVLKLFKMRSLRWQVISIIVLMAGQQLSGVNAIYYY ADQIYLSAGVNEDDVQYVTAGTGAVNVLITVCAIFVVELMGRRFLLLLGFSVCFTACCVL TGALALQDVISWMPYVSIACVISYVIGHALGPSPIPALLVTEIFLQSSRPAAYMVAGTVH WLSNFTVGLVFPFIQVGLGAYSFVIFAVICLLTTVYIFLIIPETKSKTFIEINRENLYFQ

Fv light chain variable region $\left(\mathrm{V}_{\mathrm{L}}\right)$; additional $\mathrm{C}$-terminal residues, retained after TEV cleavage, are shown in italics

ELDIVLTQSPLSLPVSLGDQASISCRSSQSIVHSNGNTYLEWYLQKPGQSPKLLIYKVSNR FSGVPDRFSGSGSGTDFTLKISRVEAEDLGVYYCFQGSHVPYTFGGGTKLEIKTSENLYF $Q$

Fv heavy chain variable region $\left(\mathrm{V}_{\mathrm{H}}\right)$; additional C-terminal residues retained after TEV cleavage, are shown in italics

LEVNLVESGGGLVQPGGSRKLSCAASGFTFSSFGMHWVRQAPEKGLEWVAHISSGSRTI DYADTVKGRFTISRDNPKNTLFLQMTSLRSEDTAIYYCARGNGYYDALDYWGQGTSVT VSSAKTTPPSVTSENLYFQ

Target identification using fluorescence-based screening methods. GLUT5 homologues were cloned into the GAL1 inducible TEV cleavable GFP-His $2 \mu$ vector pDDGFP2, transformed into the S. cerevisiae strain FGY217 (MATa, ura352 , lys $2 \Delta 201$, and pep4 $\Delta)^{40}$ and overexpressed as described previously ${ }^{41,42}$. In brief, $10 \mathrm{ml}$ S. cerevisae FGY217 cell cultures in -URA media and $0.1 \%$ glucose 
were grown at $30^{\circ} \mathrm{C}$ and expression was induced by the addition of final $2 \%$ (v/v) D-galactose at an $\mathrm{OD}_{600}$ of 0.6 . After $\sim 22 \mathrm{hrs,}$, cells were harvested, resuspended in 1 x PBS buffer and overexpression levels assessed by whole-cell GFP fluorescence ${ }^{41,42}$. Fusions with detectable expression levels were re-grown in larger 2-L culture volumes and membranes subsequently isolated. The monodispersity of expressed fusions were screened in crude dodecyl- $\beta$-Dmaltopyranoside (DDM), decyl- $\beta$-D-maltopyranoside (DM), nonyl- $\beta$-Dmaltopyranoside (NM) and n-dodecyl-N,N-dimthylamine-N-oxide (LDAO) solubilized membranes by fluorescence-detection size exclusion chromatography (FSEC) $)^{43}$ as described previously ${ }^{41}$. Out of the GLUT5 homologues screened, rat and bovine GLUT5 showed the sharpest monodispersity profiles and was the most stable after purification in detergent ${ }^{44}$.

Large-scale production and purification of rat and bovine GLUT5. For rat GLUT5, cells were harvested from $10 \mathrm{~L}$ S. cerevisiae cultures, resuspended in buffer containing 50 mM Tris-HCl pH 7.6, 1 mM EDTA, $0.6 \mathrm{M}$ sorbitol, and lysed by mechanical disruption as described previously ${ }^{42}$. Membranes were isolated by

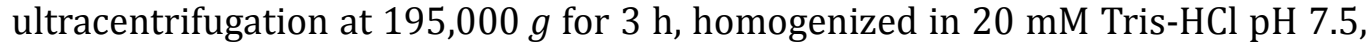
$0.3 \mathrm{M}$ sucrose, $0.1 \mathrm{mM} \mathrm{CaCl}_{2}$, frozen in liquid nitrogen and stored at $-80{ }^{\circ} \mathrm{C}$. Rat GLUT5 membranes were solubilized with 1\% DDM in equilibration buffer (EB) consisting of $1 \times$ PBS, $10 \mathrm{mM}$ imidazole, $150 \mathrm{mM} \mathrm{NaCl}, 10 \%$ glycerol. After ultracentrifugation at $195,000 \mathrm{~g}$ for $45 \mathrm{~min}$, the supernatant was incubated with $10 \mathrm{ml}$ of $\mathrm{Ni}^{2+}$-nitrilotriacetate affinity resin (Ni-NTA; Qiagen) for $2 \mathrm{~h}$ at $4{ }^{\circ} \mathrm{C}$. The resin was washed with $100 \mathrm{ml}$ of EB containing $0.05 \%$ DDM and $30 \mathrm{mM}$ imidazole, and the protein eluted in $25 \mathrm{ml}$ of EB containing $0.05 \%$ DDM and 250 $\mathrm{mM}$ imidazole. The eluted protein was incubated with TEV-His 6 protease to remove the GFP-His 8 tag and dialyzed against $20 \mathrm{mM}$ Tris- $\mathrm{HCl} \mathrm{pH} 7.5,150 \mathrm{mM}$ $\mathrm{NaCl}, 10 \%$ glycerol, $0.03 \%$ DDM. Dialyzed sample was then loaded onto a $5 \mathrm{ml}$ HisTrap column (GE Healthcare) equilibrated in dialysis buffer, and the flowthrough was collected and concentrated. The protein was further purified by size-exclusion chromatography (SEC) using a Superdex 200 column (GE Healthcare) in buffer consisting of $20 \mathrm{mM}$ Tris- $\mathrm{HCl} \mathrm{pH}$ 7.5, $150 \mathrm{mM} \mathrm{NaCl}, 0.02 \%$ 
DDM. All rat GLUT5 mutants were generated with a standard PCR-based strategy and were sub-cloned, overexpressed and purified as described for wildtype.

Protein complexes were prepared by incubation of the HisTrap-purified rat GLUT5 with the SEC-purified Fv fragment (supplemented with $0.02 \%(\mathrm{w} / \mathrm{v}$ ) DDM) at a molar ratio of $1: 1.5$ for $1 \mathrm{~h}$ on ice. The complex was subjected to SEC (Superdex 200, GE Healthcare). The SEC step was repeated twice to ensure reproducibility of crystals. Peak fractions containing rat GLUT5-Fv complex were concentrated to $\sim 10 \mathrm{mg} / \mathrm{ml}$ by ultrafiltration (Millipore, MWCO $50 \mathrm{kDa}$ ), and immediately used for crystallization experiments.

For bovine GLUT5, membranes were isolated from 40 L S. cerevisiae cultures. After membrane solubilization and ultracentrifugation (as described for rat GLUT5), the supernatant was supplemented with $55 \mathrm{mM}$ Imidazole, and incubated with $30 \mathrm{ml}$ of Ni-NTA resin for $2 \mathrm{~h}$ at $4{ }^{\circ} \mathrm{C}$. The resin was washed with $600 \mathrm{ml}$ of EB supplemented with $55 \mathrm{mM}$ imidazole and $0.03 \%$ DDM, and the protein was eluted using $125 \mathrm{ml}$ of EB containing $250 \mathrm{mM}$ imidazole and $0.03 \%$ DDM. After cleavage of GFP-His ${ }_{8}$ cleavage by TEV-His ${ }_{6}$ protease the material was re-loaded onto the same $30 \mathrm{ml} \mathrm{Ni}$-NTA resin column (no imidazole), the flowthrough was concentrated and passed through a $1 \mathrm{ml}$ Ni-NTA column for further clean up. The untagged protein was further purified by SEC in buffer containing $10 \mathrm{mM}$ Tris-HCl, pH 7.5, $150 \mathrm{mM} \mathrm{NaCl}$, and $0.09 \%$ undecyl- $\beta$-maltopyranoside (UDM $\beta$ ), and then concentrated to $\sim 4 \mathrm{mg} / \mathrm{ml}$ for crystallization experiments.

Generation of single-chain Fv (scFv) fragments. Animal experiments conformed to the guidelines outlined in the Guide for the Care and Use of Laboratory Animals of Japan and were approved by the University of Tokyo Animal Care Committee (approval no. RAC07101). Rat GLUT5-specific scFv fragment were generated essentially as previously described ${ }^{45}$. In brief, purified GLUT5 was reconstituted into liposomes containing chicken egg yolk phosphatidylcholine (egg PC; Avanti) and adjuvant lipid A (Sigma-Aldrich) by detergent removal method. Small unilamellar proteoliposomes were prepared by sonication. MRL/lpr mice were immunized with $0.1 \mathrm{mg}$ of the proteoliposome antigen three times at two-week intervals. Immunized mice were sacrificed, and 
RNA in their splenocytes was isolated and converted into cDNA via reversetranscription PCR. The $V_{L}$ and $V_{H}$ repertoire was assembled via an 18 amino acid flexible linker and cloned into the phage-display vector pComb3XSS. Biotinylated proteoliposomes were prepared by reconstituting GLUT5 with a mixture of egg PC and 1,2-dipalmitoyl-sn-glycero-3-phosphoethanolamine-N-(cap biotinyl) (16:0 biotinyl Cap-PE; Avanti), and used as binding targets for scFv-phage selection. The targets were immobilized onto streptavidin-coated paramagnetic beads (Dynabeads) or streptavidin-coated microplates (Nunc). After four rounds of biopanning, proteoliposome-targeted enzyme-linked immunosorbent assays ("liposome ELISAs") were performed on periplasmic extracts of individual colonies. Positive clones were collected and evaluated using a Biacore T100 (GE Healthcare).

Expression and purification of Fv fragment. Antibody fragments in the scFv format are undesirable for use as crystallization chaperones because they are able to intermolecularly form domain-swapped dimers, and dimer-monomer equilibrium may increase structural heterogeneity. Therefore, we used Fv fragments for crystallization trials. The $\mathrm{Fv}$ fragment were expressed in Brevibacillus choshinensis as a secreted His $_{6}$-tagged protein and purified from culture medium. The cells were grown at $30^{\circ} \mathrm{C}$ with 200 r.p.m. in $2 \mathrm{SY}$ medium (soypton $40 \mathrm{~g} / \mathrm{l}$, yeast extract $5 \mathrm{~g} / \mathrm{l}$, glucose $20 \mathrm{~g} / \mathrm{l}, \mathrm{CalCl}_{2} 0.15 \mathrm{~g} / \mathrm{l}$ ) supplemented with $50 \mathrm{mg} / \mathrm{l}$ neomycin. The cells expressing the $\mathrm{V}_{\mathrm{L}}$ and $\mathrm{V}_{\mathrm{H}}$ were initially grown separately as overnight precultures. The precultures were then combined and diluted in $2 \mathrm{SY}$ medium to give $\mathrm{OD}_{600}$ of 0.02 of each strain and grown for further 65-70 $\mathrm{h}$. The cells were removed by centrifugation at $6,000 \mathrm{~g}$ for $15 \mathrm{~min}$. The recovered culture supernatant was adjusted to a final ammonium sulfate concentration of $60 \%$ saturation, and the precipitate was pelleted, dissolved in TBS buffer (10 mM Tris- $\mathrm{HCl}, \mathrm{pH} 7.5,150 \mathrm{mM} \mathrm{NaCl}$ ), and dialyzed overnight against the same buffer. Dialyzed sample was mixed with Ni-NTA resin equilibrated with Fv1 buffer (10 mM Tris-HCl, pH 7.5, $150 \mathrm{mM} \mathrm{NaCl,} 20 \mathrm{mM}$ imidazole). Bound proteins were eluted with Fv2 buffer (10 mM Tris- $\mathrm{HCl}, \mathrm{pH} 7.5$, $150 \mathrm{mM} \mathrm{NaCl}, 250 \mathrm{mM}$ imidazole), mixed with TEV-His 6 and dialyzed overnight against TBS buffer. Cleaved His 6 tag and TEV-His 6 were removed by binding to a 
HisTrap column equilibrated with Fv1 buffer. The tag-free Fv fragment was concentrated and loaded onto a HiLoad16/60 Superdex 75 column (GE Healthcare) equilibrated with TBS buffer. Peak fractions were pooled, concentrated, flash frozen in liquid nitrogen, and stored at $-80^{\circ} \mathrm{C}$.

Transport activity of reconstituted GLUT5. The proteoliposome D-fructose uptake assay was modified from that previously described for human GLUT1 ${ }^{46}$. In brief, purified rat GLUT5 was reconstituted by the freeze-thaw/extrusion method. Crude lipids were extracted from bovine liver and sonicated to make unilamellar liposomes. $500 \mu \mathrm{L}$ of a mixture containing $10 \mu \mathrm{g}$ of purified GLUT5 and $20 \mathrm{mg}$ of liposomes in $10 \mathrm{mM} \mathrm{TrisSO}_{4}(\mathrm{pH} \mathrm{7.5)}$ was flash frozen and thawed at room temperature. Large, unilamellar proteoliposomes were prepared by extrusion (LiposoFast, Avestin; membrane pore size, $400 \mathrm{~nm}$ ). For each time point, $10 \mu \mathrm{l}$ proteoliposomes (0.4 mg lipid; $0.2 \mu \mathrm{g}$ GLUT5) was added to $10 \mu \mathrm{l}$ transport buffer containing $10 \mathrm{mM} \mathrm{TrisSO}_{4}$ and $2 \mathrm{mM} \mathrm{MgSO}_{4}(\mathrm{pH} 7.5$ ) with or

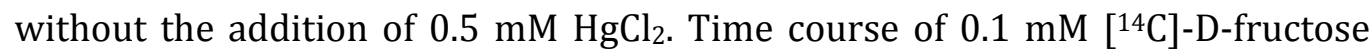
transport was measured at $25{ }^{\circ} \mathrm{C}$ at indicated time intervals and stopped by the addition of cold buffer containing $10 \mathrm{mM}^{\mathrm{TrisSO}}{ }_{4}, 2 \mathrm{mM} \mathrm{MgSO}_{4}(\mathrm{pH} 7.5)$ and 0.5 $\mathrm{mM} \mathrm{HgCl}_{2}$ and immediately filtered. Non-specific uptake was estimated with 0.1 $\mathrm{mM}\left[{ }^{14} \mathrm{C}\right]$-L-glucose. The radioactivity corresponding to the internalized substrate was measured by scintillation counting. Each experiment was performed in triplicate and data-points shown indicate average values of two technical replicates.

Substrate specificity. Unless otherwise stated the rat GLUT5 deglycosylation mutant (N50Y) used for structure determination is referred to as "wild-type like protein (WT)". Only rat GLUT5 WT and mutants showing a monodisperse peak in DDM during gel filtration were assessed for their ability to bind sugar by tryptophan fluorescence. In all experiments, either purified rat GLUT5 WT or mutants were diluted to $0.06 \mathrm{mg} / \mathrm{ml}$ in purification buffer containing $150 \mathrm{mM} \mathrm{NaCl}_{2}, 20 \mathrm{mM}$ Tris pH 7.5, 0.03\% DDM. D-fructose, Lfructose, D-glucose, D-mannose, D-xylose and D-galactose stocks, that were freshly prepared in purification buffer, were added to the diluted protein to reach a final concentration of $40 \mathrm{mM}$. After each sugar addition the sample was 
incubated at room temperature for 2 minutes before measuring tryptophan fluorescence. Measurements were performed using a Cary Eclipse Fluorescence Spectrophotometer (Agilent Technologies), with an excitation wavelength of 295 $\mathrm{nm}$ and the emission spectra recorded in the range of 300-400nm with a $5 \mathrm{~nm}$ excitation slit in a High Precision Cell Quartz Suprasil 10 x 2mm cuvette (Hellma Analytics). The emission peak of $338 \mathrm{~nm}$ was taken as an average of the four wavelengths 337.03, 337.96, 339.06 and $340 \mathrm{~nm}$. Each experiment was carried out in triplicate in the absence and presence of the well-known GLUT inhibitor $\mathrm{HgCl}_{2}(5 \mathrm{mM})^{47}$. To compare mutant binding to D-fructose, nonspecific tryptophan fluorescence quenching of $2.5 \%$ (as measured with $40 \mathrm{mM} \mathrm{L}$ fructose) was first subtracted from both wildtype and mutant quenching levels prior to calculating the final percentage of quenching relative to wildtype.

Binding analysis. D-fructose or D-glucose stocks, that were freshly prepared in purification buffer, were sequentially added to the diluted protein to a final concentration of $40 \mathrm{mM}$. After each sugar addition the sample was incubated at room temperature for 2 minutes prior to measuring. Tryptophan fluorescence measurements were performed as above. Each experiment was carried out in triplicate in the absence and presence of the known GLUT inhibitor $\mathrm{HgCl}_{2}(5$ $\mathrm{mM}$ ). The rat GLUT5 curves were fitted by non-linear regression using the software Prism. The bovine GLUT5 construct used for structural studies binds Dfructose with similar affinity as rat GLUT5 with a $K_{\mathrm{d}}$ of $5.5 \pm 0.6 \mathrm{mM}$.

Crystallization. Fv fragment. The structure of the Fv fragment was used as a search model for the molecular replacement. Crystals of the Fv fragment appeared in the well buffer at $20^{\circ} \mathrm{C}$ containing $0.1 \mathrm{M}$ CAPS-NaOH (pH 10.5), $1.6 \mathrm{M}$ $\left(\mathrm{NH}_{4}\right)_{2} \mathrm{SO}_{4}, 0.1 \mathrm{M} \mathrm{Li}_{2} \mathrm{SO}_{4}, 6.5 \mathrm{mM}$ n-nonyl- $\beta$-D-glucoside.

Rat GLUT5. Crystals of rGLUT5-Fv complex (in DDM) used for structural determination were grown at $20^{\circ} \mathrm{C}$ by hanging drop vapor diffusion. A $400-\mu \mathrm{l}$ reservoir containing 33-35 \% PEG 400, 0.12 $\mathrm{M} \mathrm{CaCl}_{2}, 0.1 \mathrm{M}$ Tris- $\mathrm{HCl}$ (pH 8.0) was equilibrated against a 2- $\mu$ d drop containing a 1:1 mixture of the complex and reservoir solution. After 2 to 3 weeks of growth, crystals were dehydrated by stepwise equilibration of the drops against $400 \mu \mathrm{l}$ reservoirs containing 
increasing concentration of PEG 400, in steps of 5\% and up to a final concentration of $70 \%$. Crystals were then flash-frozen and stored in liquid nitrogen.

Bovine GLUT5. Crystals were grown over the course of 2 to 3 weeks at $4{ }^{\circ} \mathrm{C}$ in $2 \mu \mathrm{l}$ drops (500 $\mu \mathrm{l}$ well) consisting of the well solution 31 to 35\% PEG 300, $0.125 \mathrm{M}$ HEPES pH 8.0, 0.125 M NaCl, 0.125 $\mathrm{M} \mathrm{LiSO}_{4}$, mixed first with $70 \mathrm{mM}$ HEGA-10 (4:1) and then the protein solution (1:1), and placed over the well solution diluted with water $(4: 1)$ before sealing. For freezing in liquid nitrogen, crystals were soaked for one minute in the mother liquor supplemented with 15\% PEG 300.

Data collection, structure determination and analysis. For the Fv fragment, diffraction data were collected at $100 \mathrm{~K}$ at SPring-8 beamline BL41XU (Japan) and processed using HKL2000 packages ${ }^{48}$ and the CCP4 suite ${ }^{49}$. The crystal belonged to the space group $C 2$ with two molecules per asymmetric unit. The structure was determined by molecular replacement with Molrep ${ }^{50}$ using the Fv portion of an antibody structure (PDB code: 1IGC) as a search model. Refinement were performed till the $R_{\text {free }}$ value decreased to $\sim 22 \%$ with REFMAC5. For rat GLUT5-Fv complex, diffraction data were collected at $100 \mathrm{~K}$ at SPring-8 beamline BL41XU (Japan) and processed using HKL2000 packages ${ }^{48}$ and the CCP4 suite ${ }^{49}$. The dataset used for structure determination and refinement was generated by the combination of 4 datasets from 4 independent crystals. The space group was determined to be $P 2_{1}$, with two rat GLUT5-Fv complexes per asymmetric unit. The structure was determined by molecular replacement with Molrep ${ }^{50}$ using two search models (polyalanine of the transmembrane region in the outwardfacing conformation of FucP (PDB code: 307Q), and the separately determined $1.5 \AA$ structure of the Fv fragment used in this study). Refinement were performed with PHENIX ${ }^{51}$ followed by manual examination and rebuilding of the refined coordinates using COOT52. Recently determined structures of XylE (PDB code: 4GC0) and human GLUT1 (PDB code: 4PYP) helped with the modeling. The $6 \mathrm{~N}$-terminal residues (Met1-Gln6), 22 C-terminal residues (Ser481-Gln502), and 22 residues (Asn39-Asn60) in molecule $A$ and 12 residues (Met45-Arg56) in molecule B in TM2 are not included in the structure as they did not have 
interpretable densities. For bovine GLUT5, data were collected on frozen crystals at beamline I02, Diamond Light Source (UK). The dataset used for structure determination and refinement was generated using HKL2000 by processing and scaling together two datasets collected on two different parts of the same crystal, and by correcting for anisotropy (UCLA server, http://services.mbi.ucla.edu/anisoscale/) (Extended Data Table 1 and 2 Extended Data Figure 1). The structure was solved by molecular replacement using the N-terminal and C-terminal bundles of rat GLUT5 as independent search models in PHASER, and refined using REFMAC5 and BUSTER against data up to $3.0 \AA$ resolution after anisotropy correction ${ }^{53}$, with rounds of re-building in $\mathrm{COOT}^{52}$. Final refinement was performed using PHENIX ${ }^{51}$ at $3.2 \AA$ resolution (Extended Data Figure 1). Structural alignments were performed using the align command of PYMOL software using C-alpha coordinates.

Homology modeling. Models for the outward-occluded and inward-occluded conformations were based on corresponding XylE crystal structures (4GBY and 4JA3, respectively) and were produced using Modeller 9v1254. Multiple alignment of GLUT5 homologs (Homo sapiens, Canis lupus, Rattus norvegicus, Felis catus, Gallus gallus, Anolis carolinensis) and xylE sequences were combined with structural data from the XylE structures and the GLUT5 structures using expresso mode of $t$-coffee ${ }^{55}$. The multiple alignment in combination with helical restraints corresponding to the TMs of bovine GLUT5 structure was used as an input for Modeller. For each conformation, 20 models were generated with loop optimization. The DOPE scoring function was used to select the final model. Outward-facing bovine GLUT5 was modeled with the same protocol based on the rat outward-facing GLUT5 structure and alignment of GLUT5 sequences. The four conformations (outward-open, outward-occluded, inward-occluded and inwardopen) were morphed and rendered using PyMOL (http://www.pymol.org/).

\section{References}

1 Mueckler, M. \& Thorens, B. The SLC2 (GLUT) family of membrane transporters. Molecular aspects of medicine 34, 121-138, (2013). 
2 Zhao, F. Q. \& Keating, A. F. Functional properties and genomics of glucose transporters. Current genomics 8, 113-128 (2007).

3 Simpson, I. A., Vannucci, S. J. \& Maher, F. Glucose transporters in mammalian brain. Biochemical Society transactions 22, 671-675 (1994).

4 James, D. E., Strube, M. \& Mueckler, M. Molecular cloning and characterization of an insulin-regulatable glucose transporter. Nature 338, 8387, (1989).

5 Burant, C. F., Takeda, J., Brot-Laroche, E., Bell, G. I. \& Davidson, N. O. Fructose transporter in human spermatozoa and small intestine is GLUT5. The Journal of biological chemistry 267, 14523-14526 (1992).

6 Kayano, T. et al. Human facilitative glucose transporters. Isolation, functional characterization, and gene localization of cDNAs encoding an isoform (GLUT5) expressed in small intestine, kidney, muscle, and adipose tissue and an unusual glucose transporter pseudogene-like sequence (GLUT6). The Journal of biological chemistry 265, 13276-13282 (1990).

7 Blakemore, S. J. et al. The GLUT5 hexose transporter is also localized to the basolateral membrane of the human jejunum. The Biochemical journal 309 (Pt 1), 7-12 (1995).

8 Douard, V. \& Ferraris, R. P. Regulation of the fructose transporter GLUT5 in health and disease. American journal of physiology. Endocrinology and metabolism 295, E227-237, (2008).

9 Rand, E. B., Depaoli, A. M., Davidson, N. O., Bell, G. I. \& Burant, C. F. Sequence, tissue distribution, and functional characterization of the rat fructose transporter GLUT5. The American journal of physiology 264, G11691176 (1993). 
10 Shepherd, P. R., Gibbs, E. M., Wesslau, C., Gould, G. W. \& Kahn, B. B. Human small intestine facilitative fructose/glucose transporter (GLUT5) is also present in insulin-responsive tissues and brain. Investigation of biochemical characteristics and translocation. Diabetes 41, 1360-1365 (1992).

11 Zisman, A. et al. Targeted disruption of the glucose transporter 4 selectively in muscle causes insulin resistance and glucose intolerance. Nature medicine $\mathbf{6}$, 924-928, (2000).

12 Barone, S. et al. Slc2a5 (Glut5) is essential for the absorption of fructose in the intestine and generation of fructose-induced hypertension. The Journal of biological chemistry 284, 5056-5066, (2009).

13 Douard, V. \& Ferraris, R. P. The role of fructose transporters in diseases linked to excessive fructose intake. The Journal of physiology 591, 401-414, (2013).

14 Zamora-Leon, S. P. et al. Expression of the fructose transporter GLUT5 in human breast cancer. Proceedings of the National Academy of Sciences of the United States of America 93, 1847-1852 (1996).

15 Warburg, O. On respiratory impairment in cancer cells. Science 124, 269-270 (1956).

16 Yan, N. Structural advances for the major facilitator superfamily (MFS) transporters. Trends in biochemical sciences 38, 151-159, (2013).

17 Madej, M. G., Sun, L., Yan, N. \& Kaback, H. R. Functional architecture of MFS D-glucose transporters. Proceedings of the National Academy of Sciences of the United States of America 111, E719-727, (2014).

18 Maiden, M. C., Davis, E. O., Baldwin, S. A., Moore, D. C. \& Henderson, P. J. Mammalian and bacterial sugar transport proteins are homologous. Nature 325, 641-643, (1987). 
19 Pao, S. S., Paulsen, I. T. \& Saier, M. H., Jr. Major facilitator superfamily. Microbiology and molecular biology reviews 62, 1-34 (1998).

20 Farwick, A., Bruder, S., Schadeweg, V., Oreb, M. \& Boles, E. Engineering of yeast hexose transporters to transport D-xylose without inhibition by Dglucose. Proceedings of the National Academy of Sciences of the United States of America 111, 5159-5164, (2014).

21 Deng, D. et al. Crystal structure of the human glucose transporter GLUT1. Nature 510, 121-125, (2014).

22 Sun, L. et al. Crystal structure of a bacterial homologue of glucose transporters GLUT1-4. Nature 490, 361-366, (2012).

23 Quistgaard, E. M., Low, C., Moberg, P., Tresaugues, L. \& Nordlund, P. Structural basis for substrate transport in the GLUT-homology family of monosaccharide transporters. Nature structural \& molecular biology 20, 766$768,(2013)$.

24 Wisedchaisri, G., Park, M. S., Iadanza, M. G., Zheng, H. \& Gonen, T. Protoncoupled sugar transport in the prototypical major facilitator superfamily protein XylE. Nature communications 5, 4521, (2014).

25 Iancu, C. V., Zamoon, J., Woo, S. B., Aleshin, A. \& Choe, J. Y. Crystal structure of a glucose $/ \mathrm{H}^{+}$symporter and its mechanism of action. Proceedings of the National Academy of Sciences of the United States of America 110, 17862-17867, (2013).

26 Garcia, J. C., Strube, M., Leingang, K., Keller, K. \& Mueckler, M. M. Amino acid substitutions at tryptophan 388 and tryptophan 412 of the HepG2 (Glut1) glucose transporter inhibit transport activity and targeting to the plasma 
membrane in Xenopus oocytes. The Journal of biological chemistry 267, 7770-7776 (1992).

$27 \mathrm{Li}, \mathrm{Q}$. et al. Cloning and functional characterization of the human GLUT7 isoform SLC2A7 from the small intestine. American journal of physiology. Gastrointestinal and liver physiology 287, G236-242, (2004).

28 Mueckler, M. \& Makepeace, C. Analysis of transmembrane segment 10 of the Glut1 glucose transporter by cysteine-scanning mutagenesis and substituted cysteine accessibility. The Journal of biological chemistry 277, 3498-3503, (2002).

29 Andersson, M. et al. Proton-coupled dynamics in lactose permease. Structure 20, 1893-1904, (2012).

30 Law, C. J. et al. Salt-bridge dynamics control substrate-induced conformational change in the membrane transporter GlpT. Journal of molecular biology 378, 828-839, (2008).

31 Schurmann, A. et al. Role of conserved arginine and glutamate residues on the cytosolic surface of glucose transporters for transporter function. Biochemistry 36, 12897-12902, (1997).

32 Seatter, M. J., De la Rue, S. A., Porter, L. M. \& Gould, G. W. QLS motif in transmembrane helix VII of the glucose transporter family interacts with the C-1 position of D-glucose and is involved in substrate selection at the exofacial binding site. Biochemistry 37, 1322-1326, (1998).

33 Hruz, P. W. \& Mueckler, M. M. Cysteine-scanning mutagenesis of transmembrane segment 7 of the GLUT1 glucose transporter. The Journal of biological chemistry 274, 36176-36180 (1999). 
34 Manolescu, A., Salas-Burgos, A. M., Fischbarg, J. \& Cheeseman, C. I. Identification of a hydrophobic residue as a key determinant of fructose transport by the facilitative hexose transporter SLC2A7 (GLUT7). The Journal of biological chemistry 280, 42978-42983, (2005).

35 Kasahara, T., Maeda, M., Boles, E. \& Kasahara, M. Identification of a key residue determining substrate affinity in the human glucose transporter GLUT1. Biochimica et biophysica acta 1788, 1051-1055, (2009).

36 Karpowich, N. K. \& Wang, D. N. Structural biology. Symmetric transporters for asymmetric transport. Science 321, 781-782, (2008).

37 Radestock, S. \& Forrest, L. R. The alternating-access mechanism of MFS transporters arises from inverted-topology repeats. Journal of molecular biology 407, 698-715, (2011).

38 Solcan, N. et al. Alternating access mechanism in the POT family of oligopeptide transporters. The EMBO journal 31, 3411-3421, (2012).

39 Fukuda, M. et al. Structural basis for dynamic mechanism of nitrate/nitrite antiport by NarK. Nature communications 6, 7097, (2015).

40 Kota, J., Gilstring, C. F. \& Ljungdahl, P. O. Membrane chaperone Shr3 assists in folding amino acid permeases preventing precocious ERAD. The Journal of cell biology 176, 617-628, (2007).

41 Newstead, S., Kim, H., von Heijne, G., Iwata, S. \& Drew, D. High-throughput fluorescent-based optimization of eukaryotic membrane protein overexpression and purification in Saccharomyces cerevisiae. Proceedings of the National Academy of Sciences of the United States of America 104, 1393613941, (2007). 
42 Drew, D. et al. GFP-based optimization scheme for the overexpression and purification of eukaryotic membrane proteins in Saccharomyces cerevisiae. Nature protocols $\mathbf{3}, 784-798$, (2008).

43 Kawate, T. \& Gouaux, E. Fluorescence-detection size-exclusion chromatography for precrystallization screening of integral membrane proteins. Structure 14, 673-681, (2006).

44 Sonoda, Y. et al. Benchmarking membrane protein detergent stability for improving throughput of high-resolution X-ray structures. Structure 19, 17-25, (2011).

45 Suharni, et al. Proteoliposome-based selection of a recombinant antibody fragment against the human M2 muscarinic acetylcholine receptor. Monoclonal Antibodies in Immunodiagnosis and Immunotherapy 33, 378-385 (2014).

46 Sonoda, Y. et al. Tricks of the trade used to accelerate high-resolution structure determination of membrane proteins. FEBS letters 584, 2539-2547, (2010).

47 Sarkar, H. K., Thorens, B., Lodish, H. F. \& Kaback, H. R. Expression of the human erythrocyte glucose transporter in Escherichia coli. Proceedings of the National Academy of Sciences of the United States of America 85, 5463-5467 (1988).

48 Minor, Z. O. a. W. Processing of X-ray Diffraction Data Collected in Oscillation Mode. Methods in Enzymology 276, 307-326 (1997).

49 Collaborative Computational Project, N. The CCP4 suite: programs for protein crystallography. Acta crystallographica. Section D, Biological crystallography 50, 760-763, (1994). 
50 Vagin, A. \& Teplyakov, A. Molecular replacement with MOLREP. Acta crystallographica. Section D, Biological crystallography 66, 22-25, (2010).

51 Adams, P. D. et al. PHENIX: building new software for automated crystallographic structure determination. Acta crystallographica. Section D, Biological crystallography 58, 1948-1954 (2002).

52 Emsley, P. \& Cowtan, K. Coot: model-building tools for molecular graphics. Acta crystallographica. Section D, Biological crystallography 60, 2126-2132, (2004).

53 Blanc, E. et al. Refinement of severely incomplete structures with maximum likelihood in BUSTER-TNT. Acta crystallographica. Section D, Biological crystallography 60, 2210-2221, (2004).

54 Eswar, N. et al. Comparative protein structure modeling using MODELLER. Current protocols in protein science / editorial board, John E. Coligan ... [et al.] Chapter 2, Unit 2.9., (2007).

55 Notredame, C., Higgins, D. G. \& Heringa, J. T-Coffee: A novel method for fast and accurate multiple sequence alignment. Journal of molecular biology 302, 205-217, (2000).

\section{Acknowledgements}

We are grateful to Dirk Slotboom, Alex Cameron, and Simon Newstead for discussions and comments, and James Mansfield for assistance with large-scale yeast fermentations. Data were collected at the European Synchrotron Radiation Facility, Diamond Light Source, and SPring-8 (Proposal Nos. 2011A1393, 2011B1229, 2012A1184, 2012B1253, 2013A1241, 2013B1237, 2014A1348, and 2014B1407), with excellent assistance from beamline scientists. This work was funded by the Knut and Alice Wallenberg Foundation (D.D), The Royal Society through the University Research Fellow scheme (D.D), the BBSRC (BB/G02325/1 to S.I.), the ERATO Human Receptor Crystallography Project of the Japan Science 
and Technology Agency (JST) (S.I.), by the Research Acceleration Program of the JST (S.I.), by the Targeted Proteins Research Program of the Ministry of

Education, Culture, Sports, Science and Technology (MEXT) of Japan (S.I.), and by Grants-in-Aids for Scientific Research from the MEXT (No. 22570114 to N.N.), and by the Platform for Drug Discovery, Informatics, and Structural Life Science from the MEXT (T.K.). The authors are grateful for the use of the Membrane Protein Laboratory funded by the Wellcome Trust (grant 062164/Z/00/Z) at the Diamond Light Source Limited, and The Centre for Biomembrane Research (CBR), supported by the Swedish Foundation for Strategic Research. H.J.K. was a recipient of a Human Frontiers Postdoctoral fellowship and D.D acknowledges support from EMBO through the Young Investigator Program (YIP).

\section{Author contributions}

N.N., S.I and D.D. designed the project. Cloning, expression screening and initial crystallization of rat and bovine GLUT5 was carried out by H.J.K, Y.Sonoda and D.D. Crystal optimization of bovine GLUT5 was carried out by H.J.K and G.V. Data collection, structure determination, and refinement of bovine GLUT5 was carried out by G.V. Generation of rat GLUT5 scFv fragment was carried out by N.N., Y.N., T.M., Y.N.-N., O.K.-A., H.I., T.A., T.K. and T.Hamakubo. Expression and purification of the Fv fragment was carried out by N.N., Y.N., Y.Sato, H.A. and T.Hino. Cocrystallization of rat GLUT5-Fv complex and data collection was performed by N.N., and Y.N. with assistance from T.Hino and S.I. Structure determination and refinement of rat GLUT5-Fv was carried out by T.S. Experiments for functional analysis were designed by M.K. and D.D. and carried out by M.K., D.D., S.A., A.Q. Modeling of GLUT5 was carried out by M.C. The manuscript was prepared by N.N, H.J.K, G.V, S.I and D.D. All authors discussed the results and commented on the manuscript.

The coordinates and the structure factors for bovine and rat GLUT5 have been deposited in the Protein Data Bank with entries 4YB9 and 4YBQ, respectively. Reprints and permissions information is available at www.nature.com/reprints. The authors declare no competing financial interests. Correspondence and 
request for materials should be addressed to N.N. (nnomura@mfour.med.kyotou.ac.jp), S.I (s.iwata@mfour.med.kyoto-u.ac.jp) or D.D. (ddrew@dbb.su.se).

\section{Figure legends}

Fig. 1. Structures of rat GLUT5 in the open outward-facing conformation and bovine GLUT5 in the open inward-facing conformation. a. Ribbon representation of open outward-facing rat GLUT5 (left) and open inward-facing bovine GLUT5 (right) structures, viewed in the plane of the membrane. TMs 1 and 4 and TMs 2, 3, 5 and 6 in the N-terminal TM bundle are colored in blue and light-blue, respectively. TMs 7 and 10 and TMs 8, 9, 11 and 12 in the C-terminal TM bundle are colored in red and yellow-brown, respectively. The intracellular domain helices ICH1 to ICH5 are shown in grey. b. Slab through the surface electrostatic potential of the open outward- (left) and open inward-facing (right) GLUT5 structures, as viewed within the plane of membrane, which highlight the accessibility of the sugar to the central cavity (shown as a dotted ellipse). c. Ribbon diagrams of GLUT5 viewed from the cytoplasm in the open outward(left) and inward-facing (right) conformations.

Fig. 2. The fructose-binding site of GLUT5. a. The substrate-binding site in the inward-facing bGLUT5 structure (left panel; colored as in Fig. 1) is very similar to the inward-facing hGLUT1 structure (right panel; light-grey). To facilitate comparison to rGLUT5, bGLUT5 residues are labeled with rGLUT5 numbering. For hGLUT1 only Q161 and all other residues that are different in bGLUT5 are labeled. The D-glucopyranoside moiety of bound $n$-nonyl- $\beta$-D-glucopyranoside in hGLUT1 is shown as sticks. b. D-fructose binding to GLUT5 as measured by tryptophan (Trp) fluorescence quenching (excitation $295 \mathrm{~nm}$; emission $338 \mathrm{~nm}$ ) after addition of increasing concentrations of D-fructose to WT (black squares) and to WT protein that had been previously incubated with the GLUT inhibitor $\mathrm{HgCl}_{2}$ (open circles). c. Trp fluorescence quenching for purified substrate-binding site mutants after addition of $40 \mathrm{mM}$ D-fructose (non-filled bars) relative to WT (filled bar). d. Trp fluorescence quenching after addition of either $40 \mathrm{mM}$ Dfructose or D-glucose to purified WT (black-filled bar) or Q166E (non-filled 
bars); pre-incubation with the inhibitor $\mathrm{HgCl}_{2}$ is indicated. e. Trp fluorescence quenching after addition of increasing concentrations of D-glucose to either purified Q166E (black squares), Q166E previously incubated with $\mathrm{HgCl}_{2}$ (open circles) or WT (open triangles). In all experiments errors bars, s.e.m.; $\mathrm{n}=3$.

Fig. 3. Inter-TM salt-bridges form between bundle cytoplasmic ends in the outward-facing conformation. a. Cartoon representation of GLUT5 as viewed from the cytoplasm in the outward- (left) and inward-facing (right) conformations. ICHs are not shown for clarity. TMs are colored as in Fig. 1a., and residues forming salt-bridges are shown as sticks. To facilitate comparison to rGLUT5, bGLUT5 residues are labeled with rGLUT5 numbering. Breakage of conserved salt-bridges as seen here for GLUT5 has also been predicted for XylE 23 b. Superimposition of the N- and C-terminal 6-TM bundles. Strictly conserved and pseudo symmetry related charged residues forming the salt-bridges are labeled and are shown as sticks. c. D-fructose binding as measured by Trp fluorescence quenching after incubation with $40 \mathrm{mM}$ D-fructose for purified WT GLUT5 (black-filled bar) and single alanine mutations of key acidic residues E400 and E151 that form inter-bundle salt-bridges, and G389, which is located in the hinge point of TM 10 critical for TM10 conformational change (non-filled bars). Trp fluorescence quenching by D-fructose for these mutants is displayed as a percentage of WT binding. In all experiments errors bars, s.e.m.; $n=3$.

Fig. 4. Substrate-induced gates are predominantly formed by TMs 7 and 10 in the C-terminal bundle a. Superposition of GLUT5 open outward- and inwardfacing $(*)$ structures, as viewed from the extracellular (left) and intracellular (right) side of the membrane. TMs are colored as in Fig. 1a, except inward-facing TMs $1^{*}$ and $4^{*}$ and TMs $7^{*}$ and $10^{*}$ that are coloured in orange and cyan, respectively. ICHs have been removed for clarity. b. Superimposition of the GLUT5 open outward- and inward-facing structures as viewed in the plane of the membrane. For clarity, TMs 5, 5*, 8, and 8* are not shown. Cavity-closing contacts are mostly formed by TMs $1^{*}$ and $7^{*}$ on the extracellular side in the inwardfacing conformation and by TMs 4 and 10 on the intracellular side in the outward-facing conformation. These TMs are the first TMs in each of the four 3TM repeats of the MFS fold ${ }^{16,37}$. D-xylose, as it is in the occluded-outward-facing 
XylE structure (4GBY), is shown in stick-form. With the inward movement of TM7 conserved tyrosine residues are likely to occlude the substrate from exiting, as seen for the equivalently located tyrosine residues in the substrate-occluded XylE structure $^{22}$ and as supported by D-fructose binding data (Extended Data Fig. 6d). The opening movement of TM10 to enable cytosolic substrate release has been described previously for XylE ${ }^{23,24}$ and other unrelated MFS transporters ${ }^{38,39}$ c. Interactions between hydrophobic residues between TM7 and TM10 in the outward-facing conformation (left) are lost in the inward-facing conformation (right). To facilitate comparison to rGLUT5, bGLUT5 residues are labeled with rGLUT5 numbering.

Fig. 5. Alternating-access transport mechanism in GLUT5. Schematic representation of the "rocker-switch" type movement of the $\mathrm{N}$ - and C-terminal TM bundles and of the local, gating conformational changes of TMs 7 and 10 supporting a "gated-pore" type transport mechanism in GLUT5.

\section{Extended Data and Tables}

Extended Data Table 1. Crystallographic data collection and refinement statistics.

Extended Data Table 2. Completeness of bovine GLUT5 data per resolution shell after correction for anisotropy

Extended Data Fig. 1. Anisotropy descriptors of bGLUT5 data reported by the UCLA-MBI Diffraction Anisotropy Server and 2Fo-Fc electron density maps for the bovine and rat GLUT5 structures. a. Degree of anisotropy of bGLUT5 data, resolution limits for the 3 principle axes (left), and panel illustrating steps along correction of bGLUT5 data for anisotropy (right). b Representative portions of the electron density map (1.5 $\sigma$ ) for bGLUT5 overall model (left) and a close-up of the substrate binding site (right); residues highlighted are numbered based on rGLUT5 for sake of clarity c. Electron density $(1.0 \sigma)$ for rGLUT5 showing one of the inter-bundle salt-bridge clusters that form in the open outward-facing conformation. 
Extended Data Fig. 2. Superimposition of open inward-facing bGLUT5 and hGLUT1 structures, and comparison of the substrate-binding site in bGLUT5 and inward-facing XylE a. Ribbon representation of inward-facing bGLUT5 (colored as in Fig. 1a) and inward-facing hGLUT1 (light grey) structures, as viewed in the plane of the membrane. The D-glucopyranoside moiety of the detergent molecule bound to GLUT1 ( $n$-nonyl- $\beta$-D-glucopyranoside $(\beta-N G)$ ) is shown as sticks. Density for ICH5 at the C-terminus is missing in both hGLUT1 and bGLUT5 inward-facing structures and highlighted with the dotted ellipse. The beginning of TM1 kinks further outwards in the bGLUT5 structure compared to hGLUT1 and residues 1 to 18 could not be built. The r.m.s.d. (root mean square deviation) after superposition of the two structures is $1.12 \AA$ for 364 pairs of $\mathrm{C} \alpha$ atoms?(see Methods). b The substrate-binding in the inward-facing bGLUT5 structure (coloured as in Fig. 1), is very similar to that seen in inward-facing XylE (4JA4) structure (shown in light-grey). Only non-conserved residues and the equivalent glutamine to Q166 are labeled for XylE.

Extended Data Fig. 3. Structure of the rat GLUT5-Fv complex. a. Cartoon representation of the complex between rGLUT5 (grey) and 4D111Fv (heavychain variable region $\left(\mathrm{V}_{\mathrm{H}}\right)$ is in blue; light-chain variable region $\left(\mathrm{V}_{\mathrm{L}}\right)$ is in red). 4D111Fv binds to the cytoplasmic domain of GLUT5, including ICH2 (residues 226, 230, 234), the loop between ICH2 and ICH3 (residues 238, 240, 241), and ICH3 (residue 243), with $\sim 848 \AA^{2}$ of buried surface area at the interface. b. Packing of the rat GLUT5-Fv complex molecules in the crystal. The unit cell is represented as green lines.

Extended Data Fig. 4. Sequence alignment of rat GLUT5 (rGLUT5), bovine GLUT5 (bGLUT5), human GLUT5 and GLUT7 (hGLUT5, hGLUT7), human GLUT1-4 (hGLUT1-4), Saccharomyces cerevisiae (HXT7), Plasmodium falciparum (PfHT1), Arabidopsis thaliana (GIcT) and E. coli XylE. Secondary structure elements of rat GLUT5 are indicated above the alignment, and coloured as in Fig. 1a. Strictly conserved residues are highlighted in black-filled boxes, and highly conserved residues are shaded in grey. Green boxes highlight central cavity residues that are specific to GLUT5 and red boxes highlight those that are conserved among GLUTs. Purples boxes highlight residues forming the salt- 
bridges between cytosolic TM segments. A blue box (TM5) highlights Gln166, whose mutation to glutamic acid, as present in GLUT7, weakens D-fructose binding but supports strong D-glucose binding in rGLUT5. The brown box (TM8) highlights Glu336 that is conserved across all the GLUTs and replaced with glutamic acid in XylE. Red bars underneath the alignment indicate the sugar porter (SP) family motifs ${ }^{18,19}$. Note that because bGLUT5 and hGLUT5 have an additional amino acid at position 8 their numbering differs from rGLUT5 by 1 amino acid. For clarity, bGLUT5 residues are labeled using rGLUT5 numbering.

Extended Data Fig. 5. D-fructose binding monitored by tryptophan fluorescence quenching. a. Cartoon representation of the outward-facing rGLUT5 structure, as viewed from the plane of the membrane with the coloring as shown in Fig. 1a. Atoms in all tryptophan residues are shown as spheres and tryptophan W419, whose fluorescence is quenched by substrate, is labeled. b. Emission fluorescence spectra for purified deglycosylated rGLUT5 wildtype like mutant N50Y (referred to as "WT"), shown in the range of 320-360 nm with an excitation wavelength of $295 \mathrm{~nm}$ after the addition of $40 \mathrm{mM}$ D-fructose (top), and $40 \mathrm{mM}$ L-fructose (bottom). Emission fluorescence spectra for purified WT protein that had been previously incubated with the inhibitor $\mathrm{HgCl}_{2}$ is also shown for D-fructose (middle). c. Tryptophan fluorescence quenching (excitation 295 $\mathrm{nm}$; emission $338 \mathrm{~nm}$ ) after incubation of purified rGLUT5 N50Y with either 40 mM D-fructose (black-filled bar) or L-fructose, D-glucose, D-mannose, D-xylose or D-galactose as labeled (open bars). Tryptophan fluorescence quenching for purified WT protein that had been previously incubated with the inhibitor $\mathrm{HgCl}_{2}$ is also shown for D-fructose (non-filled bar) d. As in c., rGLUT5 with a single tryptophan residue (W419), which contains the following mutations: N50Y, W70F, W191F, W239F, W265F, W275F, W338F and W370F. No tryptophan quenching was observed for D-fructose ( $5 \mathrm{mM} \mathrm{HgCl}_{2}$ ), L-fructose, D-glucose or Dgalactose. In all experiments errors bars, s.e.m.; $\mathrm{n}=3$.

Extended Data Fig. 6. Substrate-specificity in GLUT5. a. Time-dependent uptake of D-[14C]-fructose by rGLUT5 wildtype (open squares and triangles) and the deglycosylated mutant N50Y (filled squares and triangles) in proteoliposomes incubated with or without the inhibitor $\mathrm{HgCl}_{2}$ as labeled. Non- 
specific uptake was estimated with $0.1 \mathrm{mM} \mathrm{L-}\left[{ }^{14} \mathrm{C}\right]$-Glucose for wildtype (filled circles) and the N50Y mutant (open circles). In all experiments errors bars represent a spread of duplicates. Inset shows SDS-PAGE analysis of the purified rat GLUT5 wildtype and the deglycosylated N50Y mutant. b. Tryptophan fluorescence quenching (excitation $295 \mathrm{~nm}$; emission $338 \mathrm{~nm}$ ), after incubation of purified rat GLUT5 mutant (N50Y, W70F, W191F, W239F, W265F, W275F, W338F, W370F) that contains one single tryptophan residue, W419, with increasing concentrations of D-fructose (filled squares) and to the protein previously incubated with the inhibitor mercury chloride (open circles). c. Slab through the surface of the outward-facing rGLUT5 structure as viewed in the plane of membrane. The structure of substrate-bound XylE structure was further superimposed onto rGLUT5 and is shown here as a grey ribbon. In XylE, Trp392 (Trp388 in hGLUT1) is located at the bottom of the cavity (spheres; magneta) and coordinates D-xylose (stick form; yellow). In GLUT5, the equivalent residue is an alanine, making the cavity deeper. d. D-fructose binding as measured by tryptophan fluorescence quenching (excitation $295 \mathrm{~nm}$; emission $338 \mathrm{~nm}$ ) after incubation with $40 \mathrm{mM}$ D-fructose for WT (open bar), and TM7 mutations of Ile295 (interacts with TM10 residues) and Tyr296 and Tyr297 residues. Equivalently located tyrosine residues in XylE occlude the sugar-binding site from the outside 22 . Fluorescence quenching for the mutants are displayed as a percentage of total WT binding. In all experiments errors bars, s.e.m.; $n=3$.

Extended Data Fig. 7 The intracellular helical domain (ICH) a. Cytoplasmic view of the ICH domain after superposition of the open, outward-facing rGLUT5Fv (grey) and outward-facing occluded E. coli XylE (teal) (4GBY) structures. b. In the outward-facing GLUT5 structure ICH1-3 are linked together by several saltbridges (side chains are labeled and shown as sticks in yellow). In contrast, no polar interactions are formed between ICH5 and either ICH1-3 or cytoplasmic ends of N-terminal TM bundle helices. A salt-bridge forms (dotted line in magenta), however, between Glu225 in ICH3 and Arg407 in TM 11, which also forms part of the inter-bundle salt-bridge network (side chains are labeled and shown as sticks in cyan). c. In the inward-facing GLUT5 structure, this interbundle salt-bridge network is not formed, because the cytoplasmic ends of the $\mathrm{N}$ - 
and C-terminal bundle have moved apart; consistently, the ICH domain functional role is proposed to act as a scaffold domain that further helps to stabilize the outward-facing conformation ${ }^{21}$.

Extended Data Fig. 8. Access to the central cavity and substrate-binding site is gated by TM7 on the outside and TM10 on the inside. a. Superposition of outward-facing open GLUT5 and outward-facing occluded E. coli XylE (4GBY) structures. The TM numbering for outward-facing occluded XylE has an additional asterisk “*”. The inward-facing GLUT5 structure is colored as in Fig. 1a and that of XylE in grey. The bound D-xylose in stick form in green. The r.m.s.d. is $1.38 \AA ̊$ for 290 pairs of C $\alpha$ atoms (see Methods). b. Superposition of inward-open GLUT5 and inward-occluded E. coli XylE structure (4JA3) with coloring and annotation as described in a. The r.m.s.d. is $1.80 \AA$ for 274 pairs of $\mathrm{C} \alpha$ atoms (see Methods). The bound D-xylose in 4GBY is represented in stick form in green. The ICH domain is not shown for clarity. c. Superposition of inward-facing open GLUT5 and inward-facing open XylE (4JA4) structures as viewed from the cytoplasmic side with coloring and annotation as described in a. The ICH domain is not shown for clarity. The r.m.s.d. is $1.70 \AA$ for 273 pairs of $\mathrm{C} \alpha$ atoms (see Methods). 
2 京都大学 a

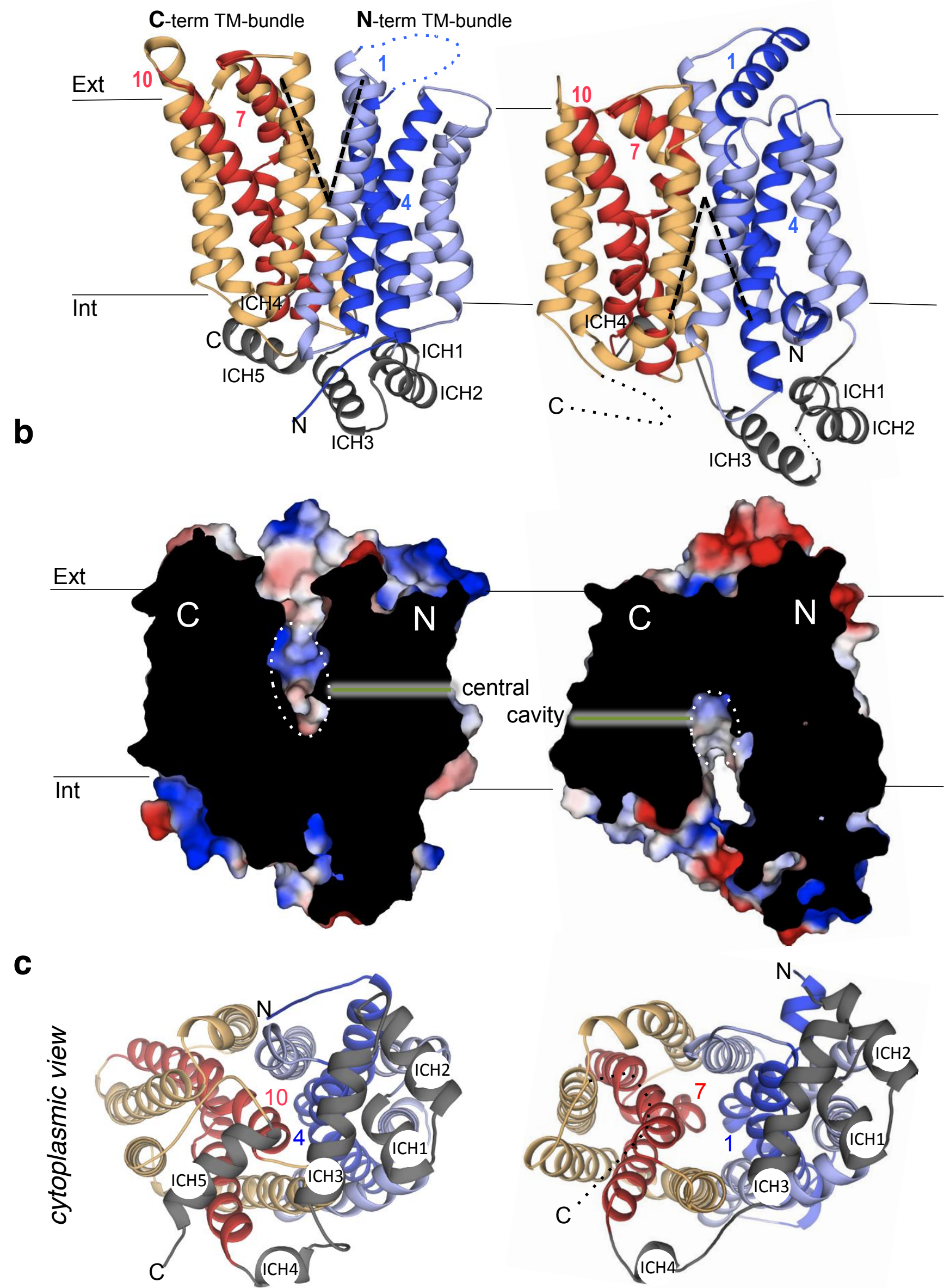


a

extracellular view

C-bundle

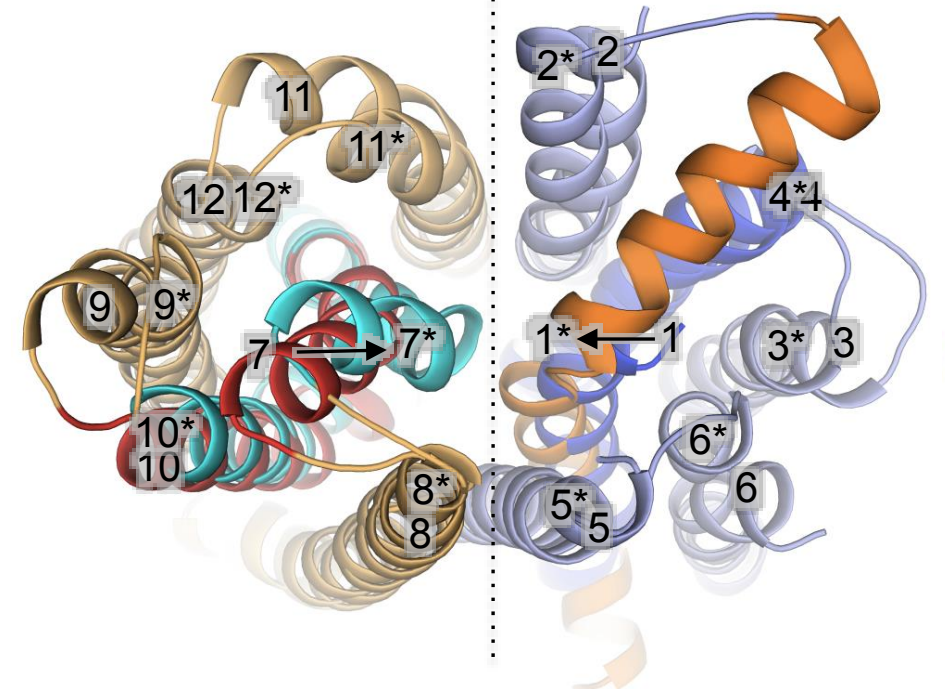

b

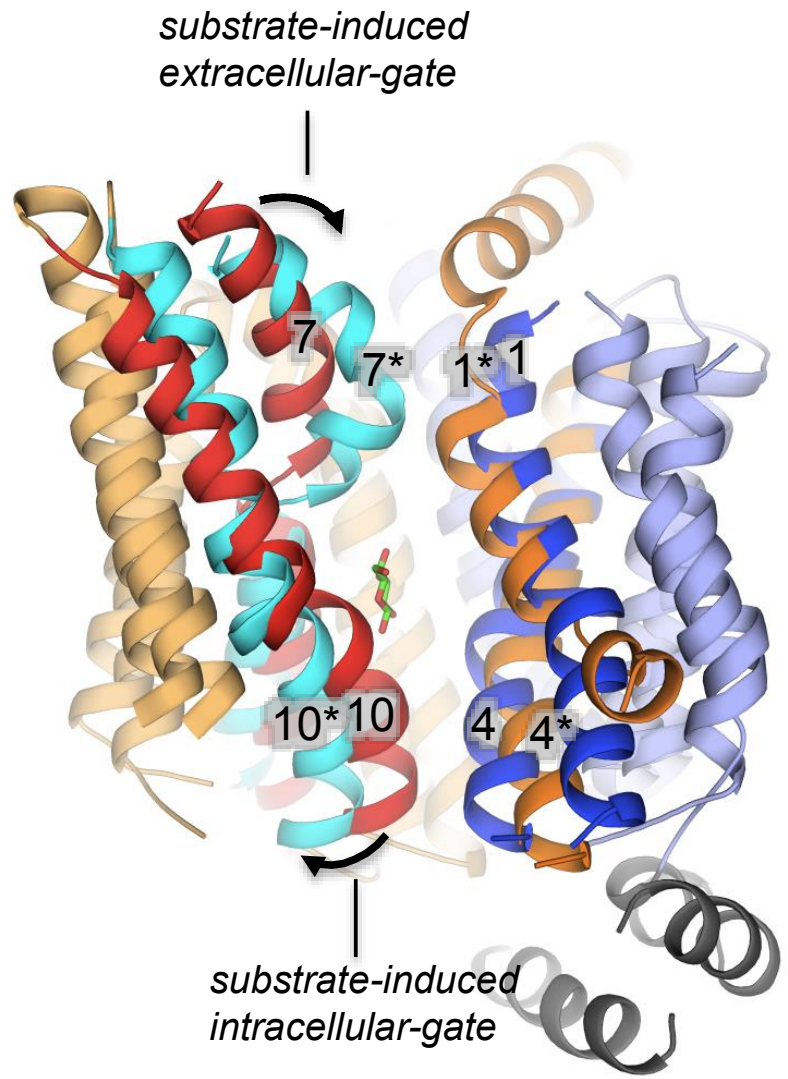

intracellular view

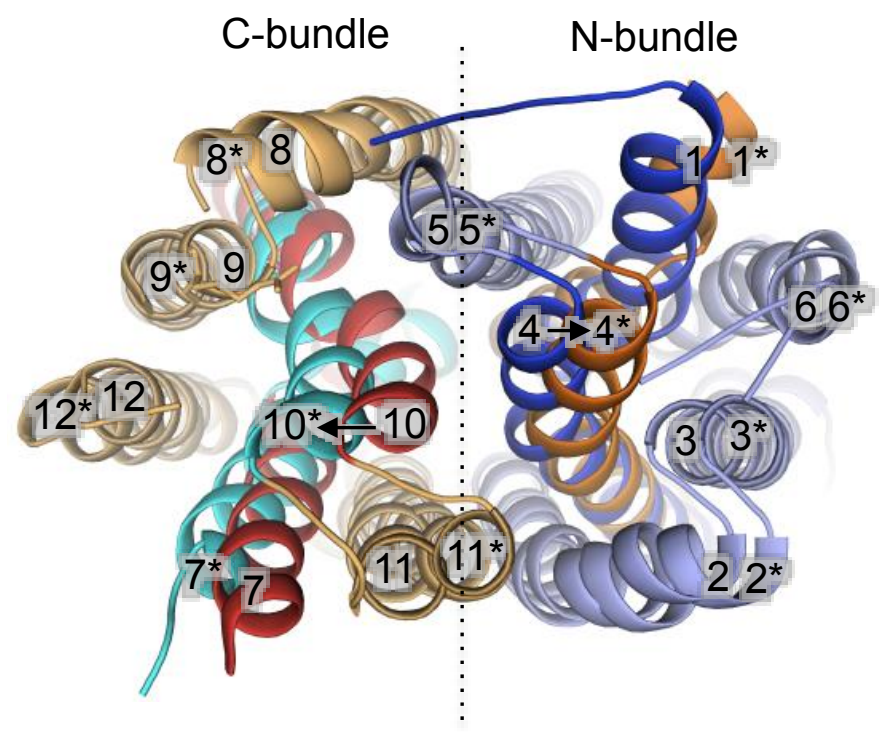

C outward-facing

inward-facing

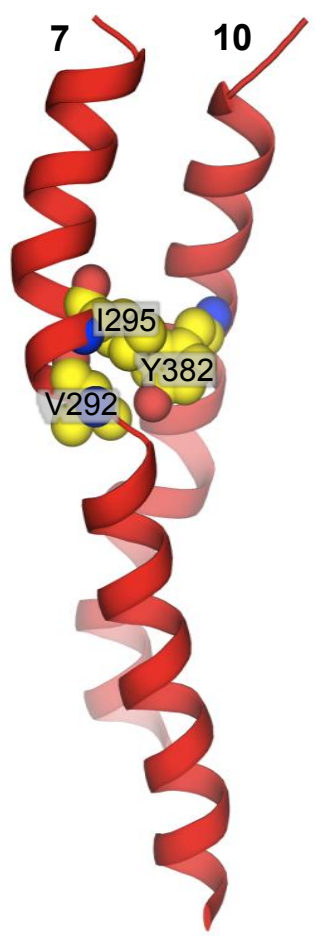

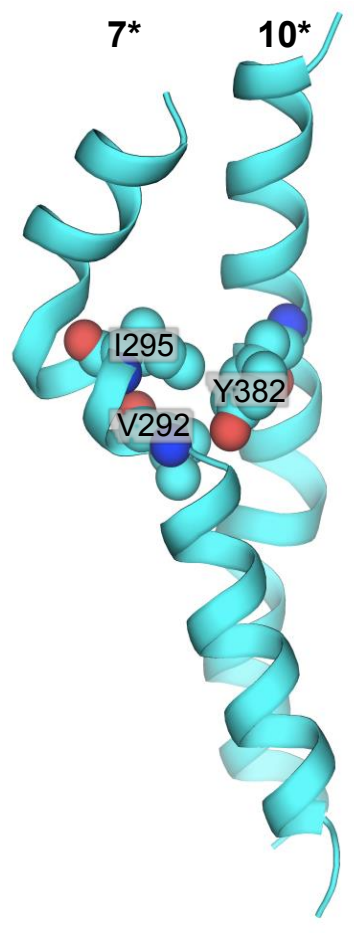


outward-open
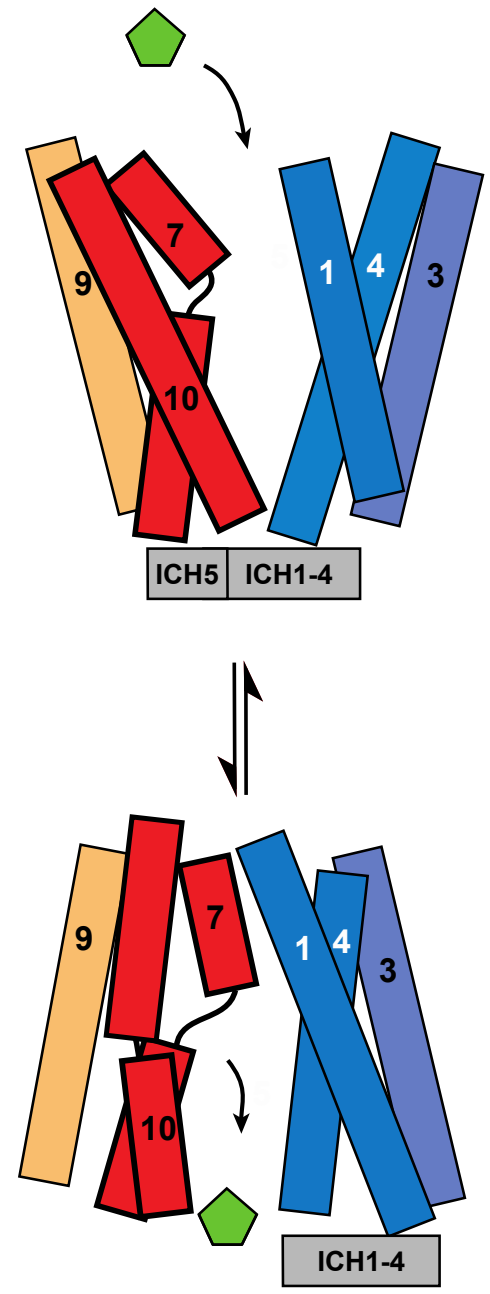

inward-open outward-occluded
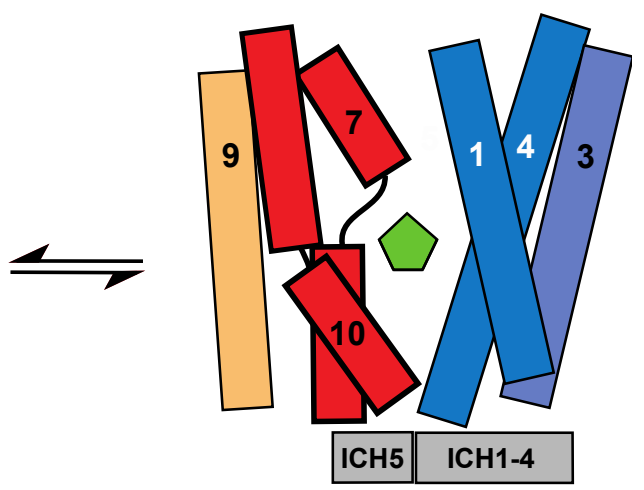

$\|$
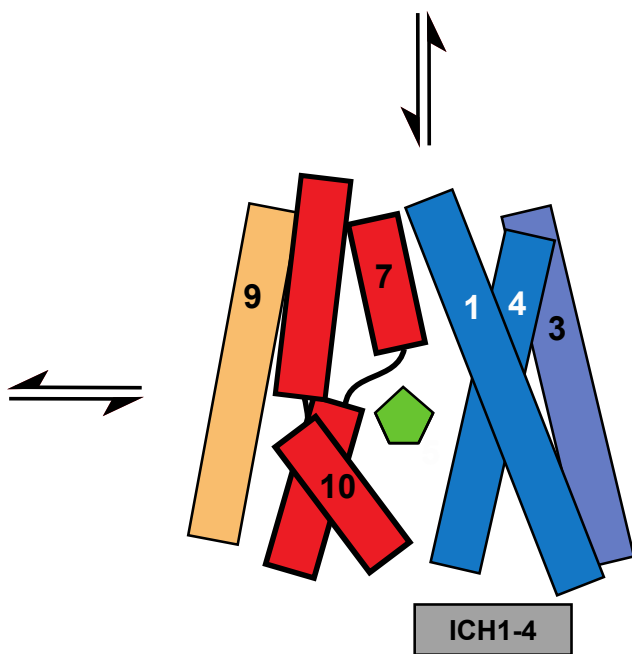

inward-occluded 
Extended Data Table 1. Data collection and refinement statistics.

\begin{tabular}{|c|c|c|}
\hline & rat GLUT5-Fv & bovine GLUT5 ${ }^{\#}$ \\
\hline \multicolumn{3}{|l|}{ Data collection } \\
\hline Space group & $P 2_{1}$ & $P 2_{1} 2_{1} 2_{1}$ \\
\hline \multicolumn{3}{|l|}{ Cell dimensions } \\
\hline$a, b, c(\AA)$ & $76.78,151.54,106.40$ & $74.61,112.15,139.57$ \\
\hline$\alpha, \beta, \gamma\left({ }^{\circ}\right)$ & $90.00,97.25,90.00$ & $90,00,90.00,90.00$ \\
\hline Resolution (A) & $50-3.27(3.39-3.27)$ & $100-3.00(3.11-3.01)^{*}$ \\
\hline$R_{\mathrm{sym}}$ or $R_{\text {merge }}$ & $21.5(>100)$ & $10.06(>100)$ \\
\hline $\mid / \sigma l$ & $11.6(1.5)$ & $11.21(0.86)$ \\
\hline Completeness (\%) & $100(100.0)$ & $99.4(93.9)$ \\
\hline Redundancy & $13.0(13.0)$ & $10.2(8.6)$ \\
\hline \multicolumn{3}{|l|}{ Refinement } \\
\hline Resolution $(\AA)$ & $50-3.27(3.5-3.27)$ & $33.3-3.2(3.31-3.20)$ \\
\hline No. reflections (Rfree set) & 38017 (3355) & 13346 (1331) \\
\hline$R_{\text {workt }} R_{\text {free }}$ & $24.2 / 28.8(35.4 / 37.5)$ & $23.6 / 25.8(32.8 / 36.5)$ \\
\hline \multicolumn{3}{|l|}{ No. atoms } \\
\hline Protein & 10657 & 3382 \\
\hline \multicolumn{3}{|l|}{ B-factors } \\
\hline Protein & 157.0 & 149.2 \\
\hline \multicolumn{3}{|l|}{ R.m.s deviations } \\
\hline Bond lengths $(\AA)$ & 0.004 & 0.003 \\
\hline Bond angles $\left({ }^{\circ}\right)$ & 0.97 & 0.90 \\
\hline \multicolumn{3}{|l|}{ Ramachandran plot (\%) } \\
\hline Favored & 98.0 & 94.5 \\
\hline Outliers & 0 & 0 \\
\hline
\end{tabular}

${ }^{\#}$ Data were obtained by scaling together two datasets collected on the same crystal

${ }^{*}$ Highest resolution shell used in the final refinement is shown in parenthesis. 
Extended Data Table 2

Extended Data Table 2. Completeness of bovine GLUT5 dataset after correction for anisotropy

\begin{tabular}{|c|c|}
\hline Resolution range $(\AA)$ & Completeness (\%) \\
\hline $100.0-8.10$ & 95.5 \\
\hline $8.10-6.43$ & 100.0 \\
\hline $6.43-5.62$ & 99.9 \\
\hline $5.62-5.10$ & 99.9 \\
\hline $5.10-4.74$ & 100.0 \\
\hline $4.74-4.46$ & 99.7 \\
\hline $4.46-4.24$ & 99.6 \\
\hline $4.24-4.05$ & 100.0 \\
\hline $4.05-3.90$ & 78.4 \\
\hline $3.90-3.76$ & 53.4 \\
\hline $3.76-3.64$ & 40.6 \\
\hline $3.64-3.54$ & 33.4 \\
\hline $3.54-3.45$ & 26.3 \\
\hline $3.45-3.36$ & 21.2 \\
\hline $3.36-3.29$ & 15.4 \\
\hline $3.29-3.22$ & 13.1 \\
\hline $3.22-3.15$ & 9.4 \\
\hline $3.15-3.09$ & 6.0 \\
\hline $3.09-3.04$ & 3.5 \\
\hline $3.04-3.00$ & 1.7 \\
\hline
\end{tabular}


a

$$
\begin{aligned}
& \text { bGLUT5 Your data has SEVERE anisotropy }
\end{aligned}
$$

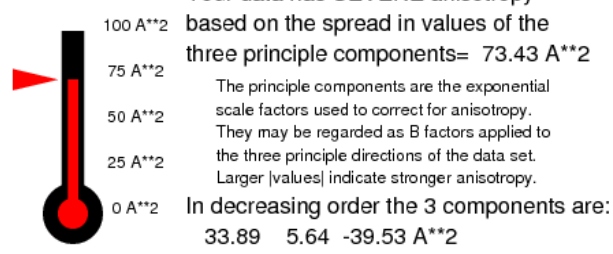

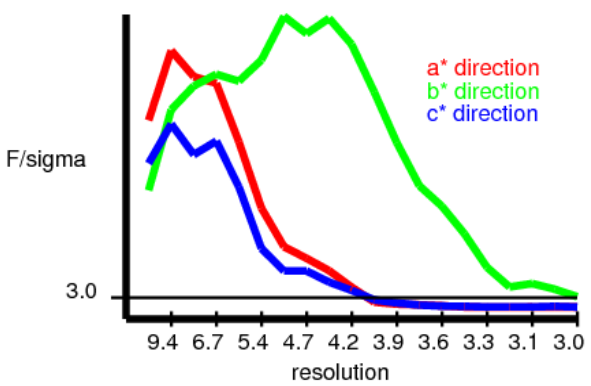

b

The recommended resolution limits along $\mathrm{a}^{*}, \mathrm{~b}^{*}, \mathrm{c}^{*}$ are

$$
\begin{aligned}
& \text { 4.0 Ang 3.0 Ang 4.0 Ang } \\
& \text { These are the resolutions at which F/sigma } \\
& \text { drops below an arbitrary cutoff of } 3.0
\end{aligned}
$$

Extended Data Fig. 1

24188 reflections were in the initial data set. 10597 were discarded because they fell outside the specified ellipsoid with dimensions 1/4.0, 1/3.0, 1/4.0 A along $\mathrm{a}^{*}, \mathrm{~b}^{*}, \mathrm{c}^{*}$, respectively. These discarded reflections had an average $\mathrm{F} /$ sigma of 2.41 .

13591 reflections remain after ellipsoidal truncation. Anisotropic scale factors were then applied to remove anisotropy from the data set. Lastly, an isotropic $B$ of $-81.00 \mathrm{~A}$ anisotropic scaling. The following pseudo precession images illustrate the individual steps.
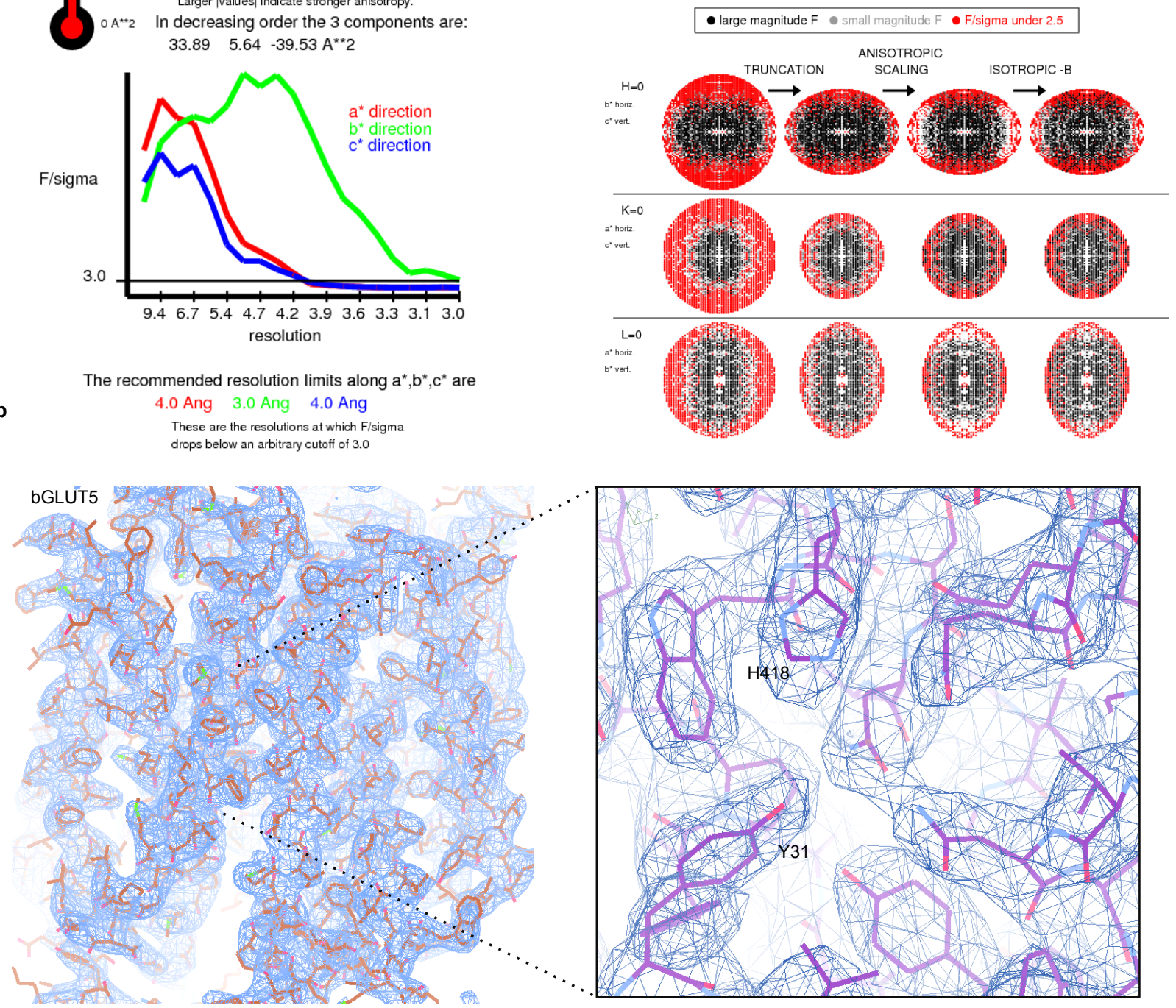

c

rGLUT5

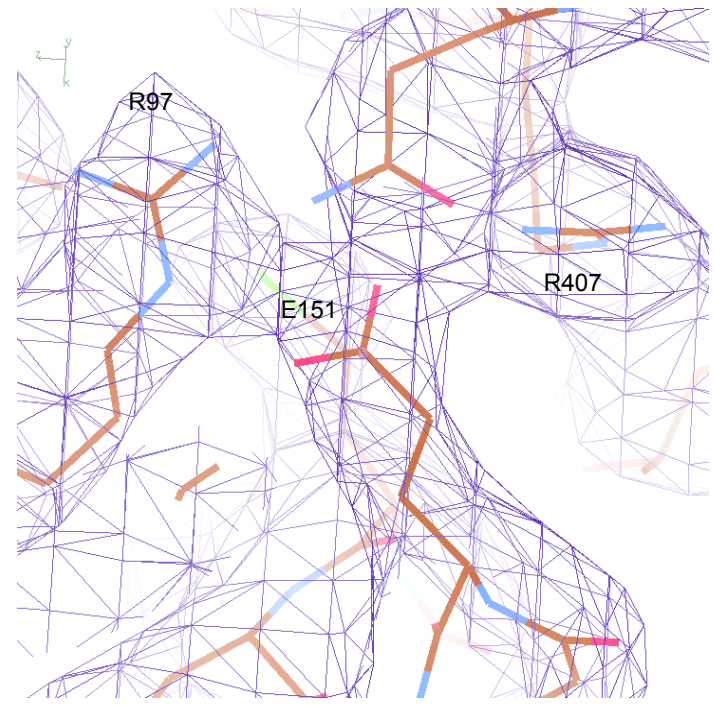


Extended Data Fig. 2

a

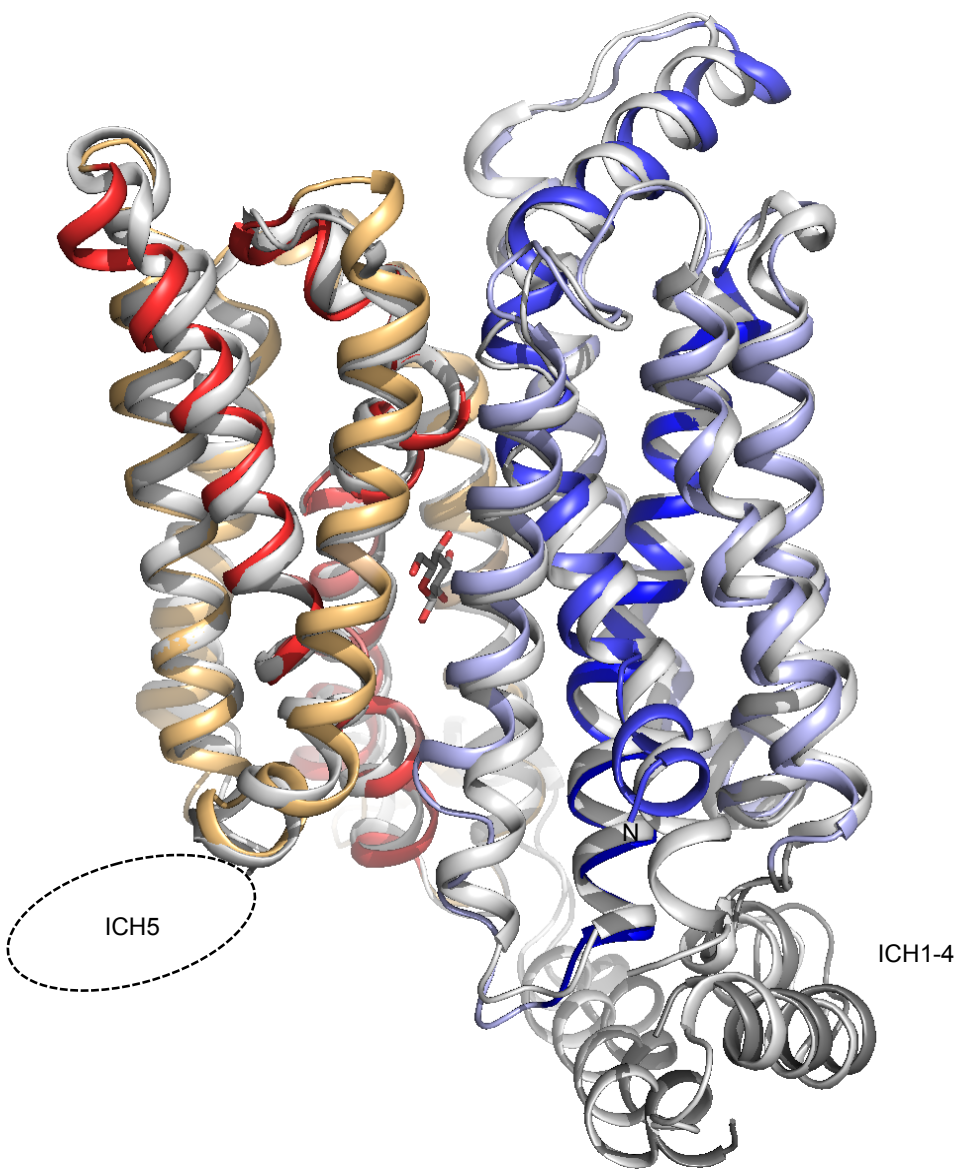

b GLUT5 and XyIE (grey)

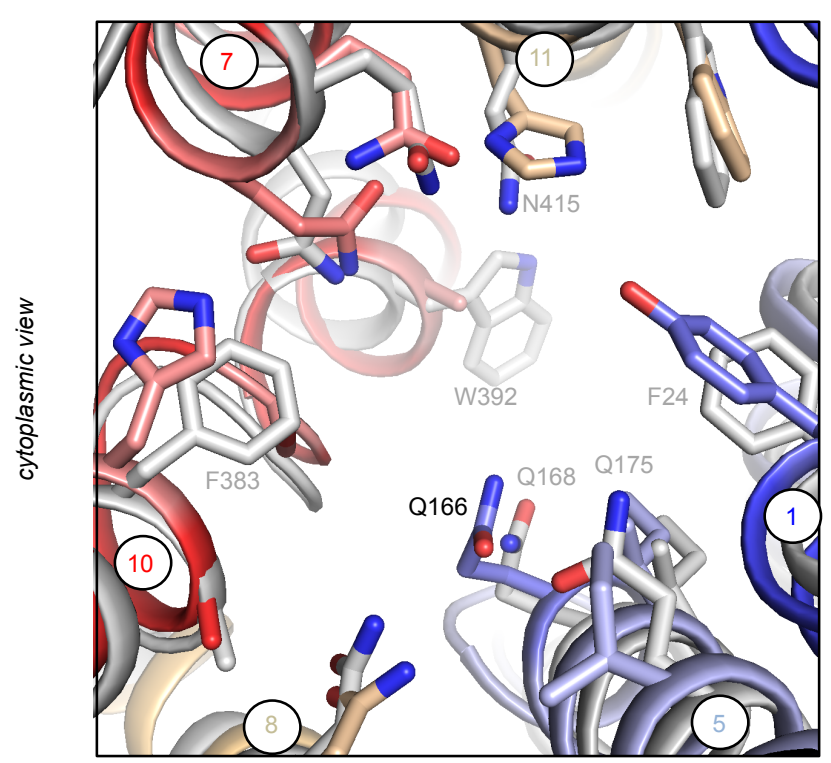


Extended Data Fig. 3

a

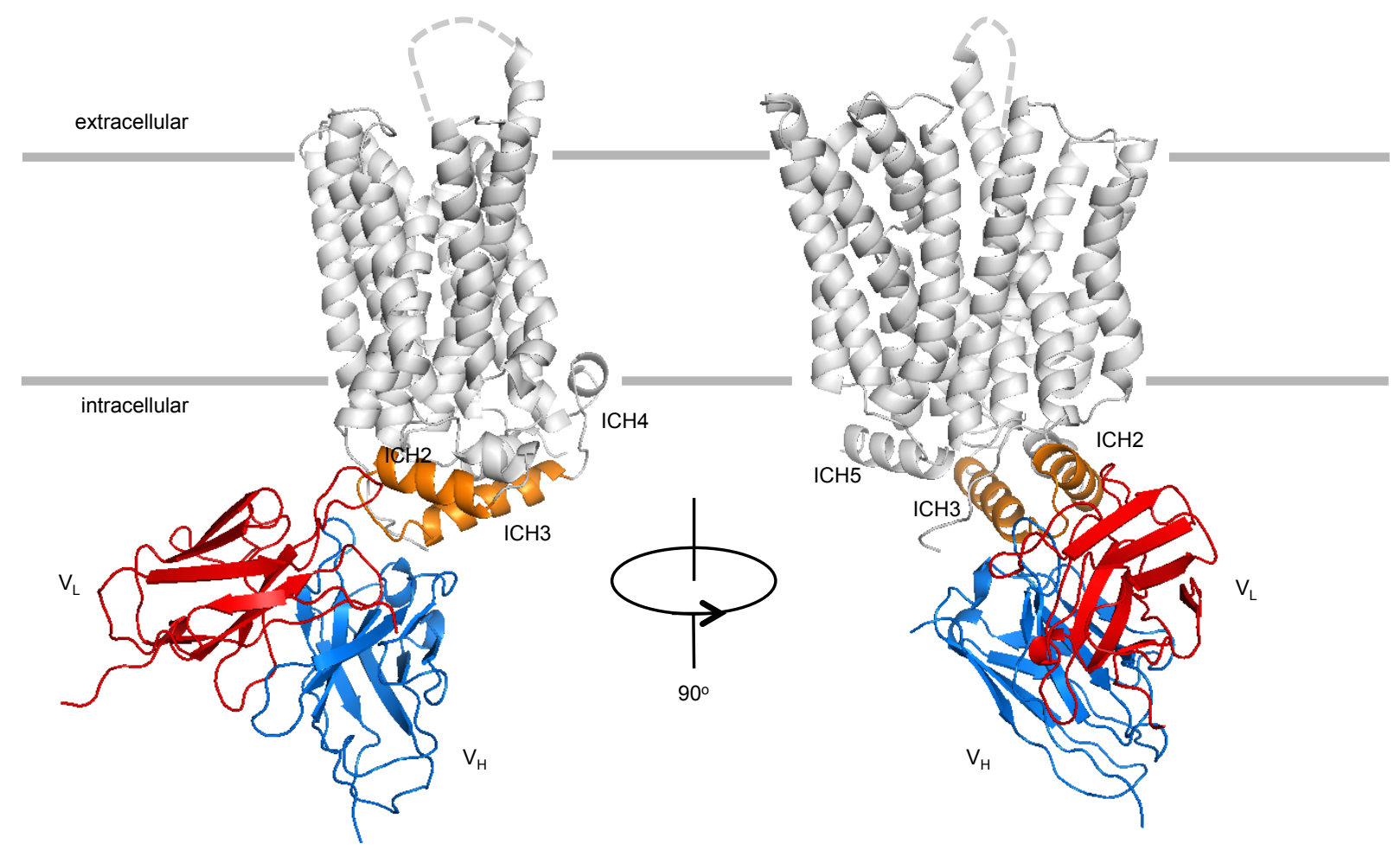

b

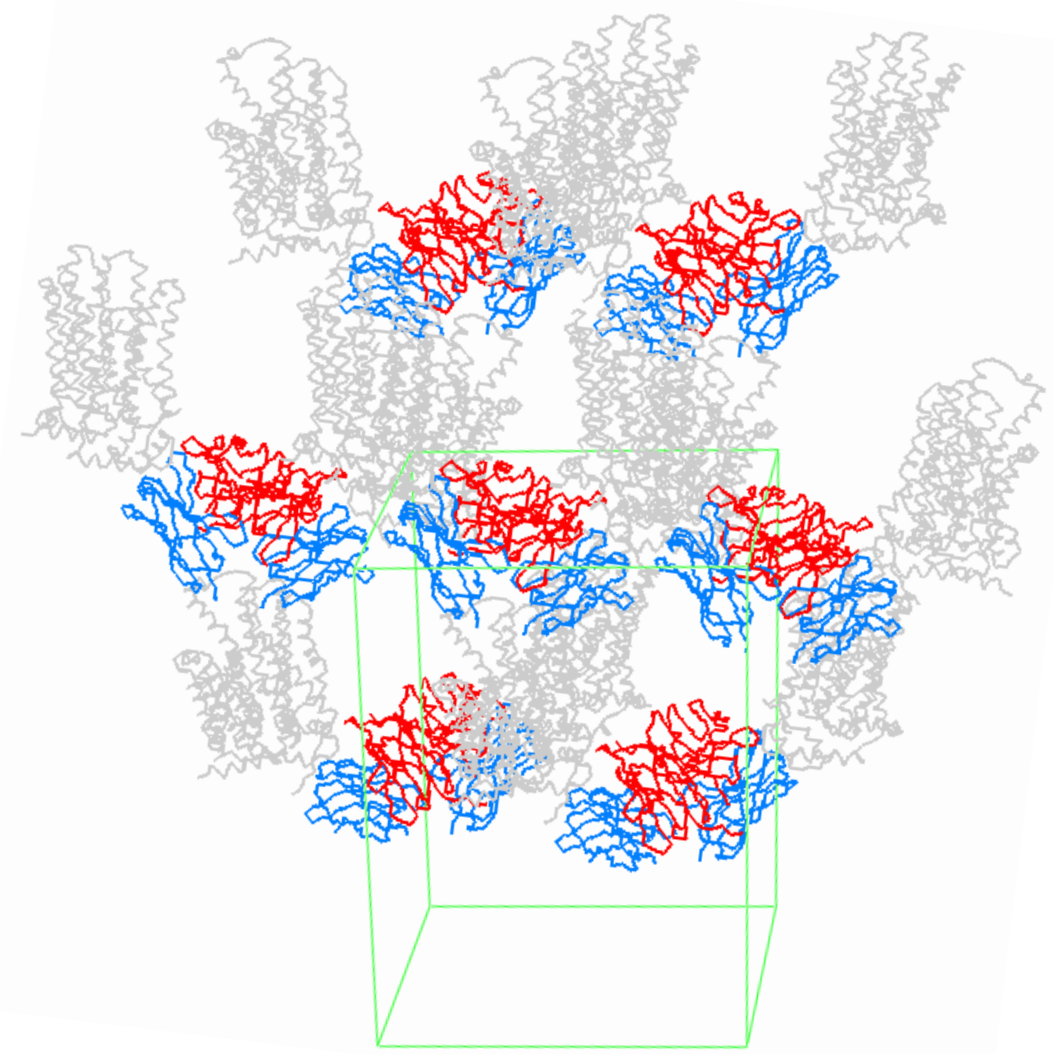


Extended Data Fig. 4

$\begin{aligned} \text { rGLUT5 } & 1 \\ \text { bGLUT5 } & 1 \\ \text { hGLUT5 } & 1 \\ \text { hGLUT7 } & 1 \\ \text { hGLUT1 } & 1 \\ \text { hGLUT2 } & 1 \\ \text { hGLUT3 } & 1 \\ \text { hGLUT4 } & 1 \\ \text { HXT7 } & 1 \\ \text { PfHT1 } & 1 \\ \text { G1CT } & 1 \\ \text { XYIE } & 1\end{aligned}$

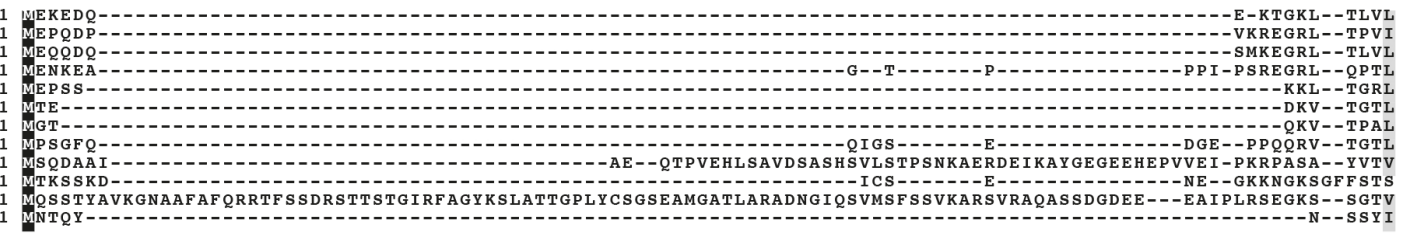

TM1

TM2

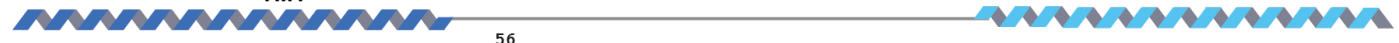

rGLUT5
bGLUT5
hGLUT5
hGLUT7
hGLUT1
hGLUT2
hGLUT
hGLUT4
HXT7
PfHT1
GICT
XY1E

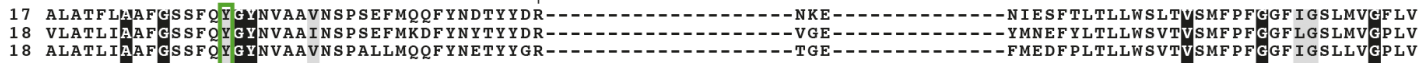
18 ALATLIAAFGSSFQY GYNVAAVNSPALLMQQFYNETYYGR---

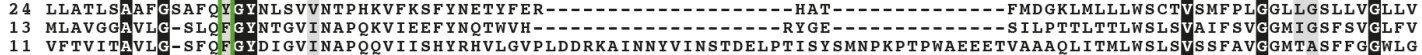
11 IFAITVATIG-SFOFGYNTGVINAPEKI IKEF INKTLTD--

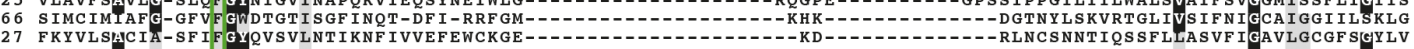

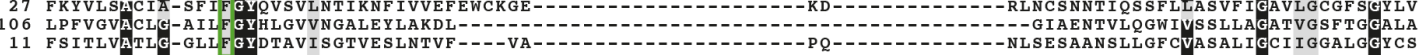

\section{TM3}

TM4

TM5

-

rGLUT5
bGLUT5
hGLUT5
hGLUT7
hGLUT1
hGLUT2
hGLUT3
hGLUT4
HXT7
PEHT1
GICT
XY1E

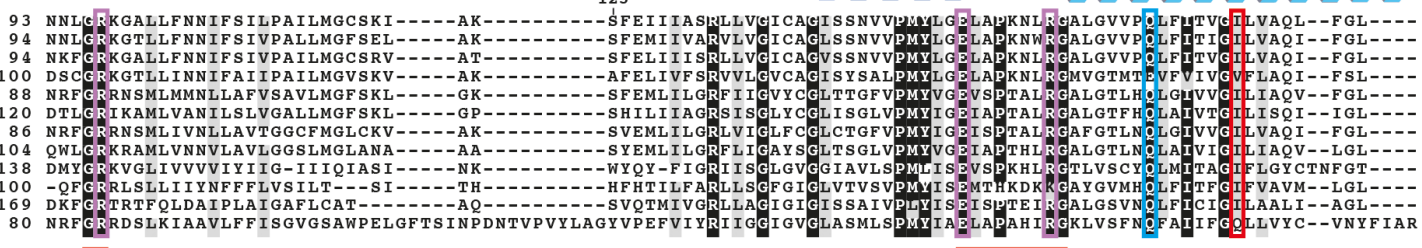

TM6

ICH1

ICH2

$\mathrm{ICH} 3$

$\mathrm{ICH} 4$

rGLUT5
bGLUT5
hGLUT5
hGLUT7
hGLUT1
hGLUT2
hGLUT3
hGLUT4
HXT7
PAHT1
GICT
XY1E

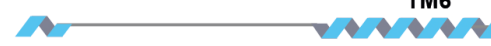

213

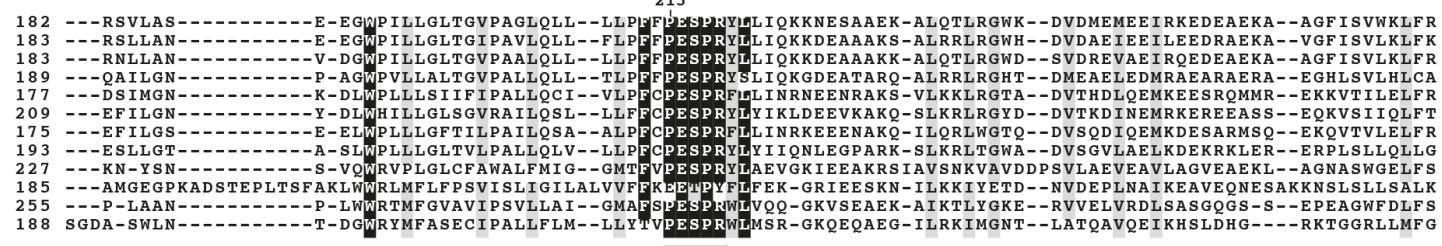

TM7a

TM7b

TM8

TM9

rGLUT5
bGLUT5
hGLUT5
hGLUT7
hGLUT1
hGLUT2
hGLUT3
hGLUT4
HXT7
PATT1
GICT
XYIE

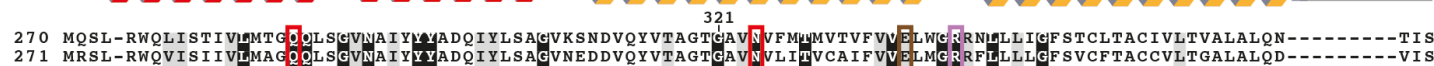

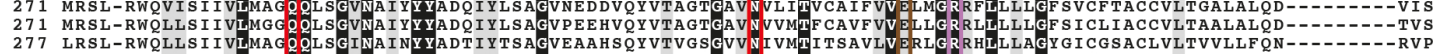

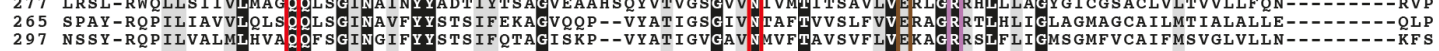

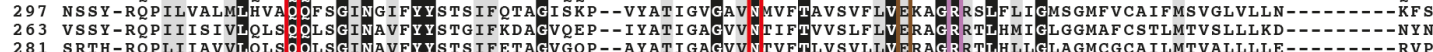

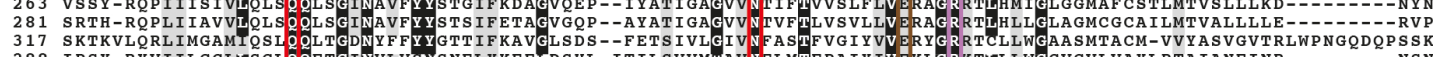

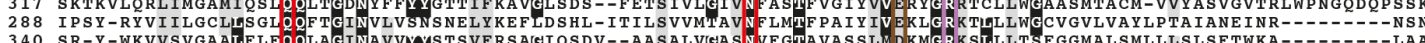

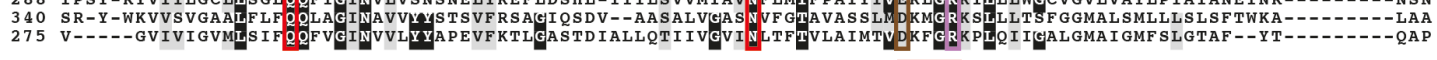

TM10

TM11

TM10

ICH5

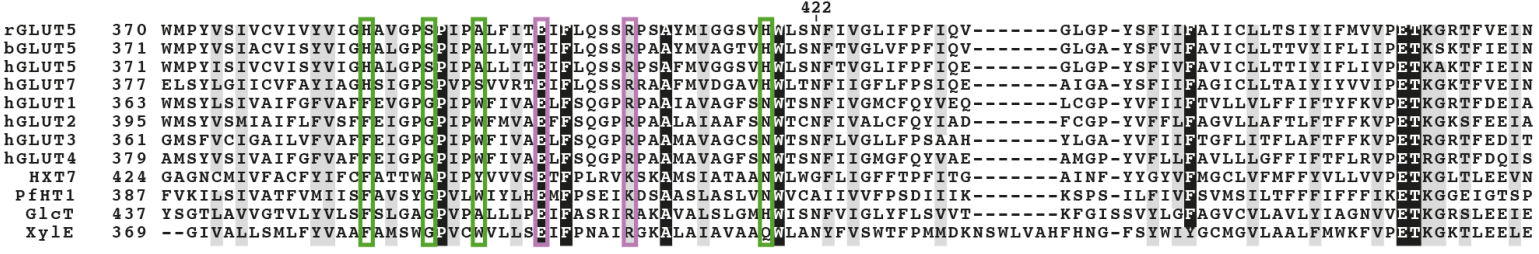

Dar-

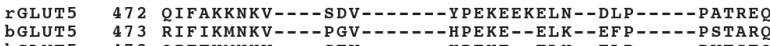

hGLUT5 473 QIFTKMNKV---SEV----YPEKE--ELK--ELP-----PVTSEQ

465 SGFROGGA-----SOS-----DKTPE--ELF--H-- - PIGADSOV

hGLUT2 497 AEFQKKSGS----AHR------ - PKAA-VEMK---

hGLUT 3463 RAFEGQAHG----ADR------SGKDGVMEMN--SIEP--AKETTTNV

LGLUT4 481 AAFHRTPSL- 48 -

PfHT1 489 YITM--

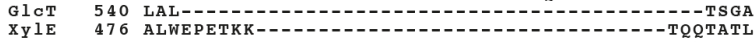


a

b

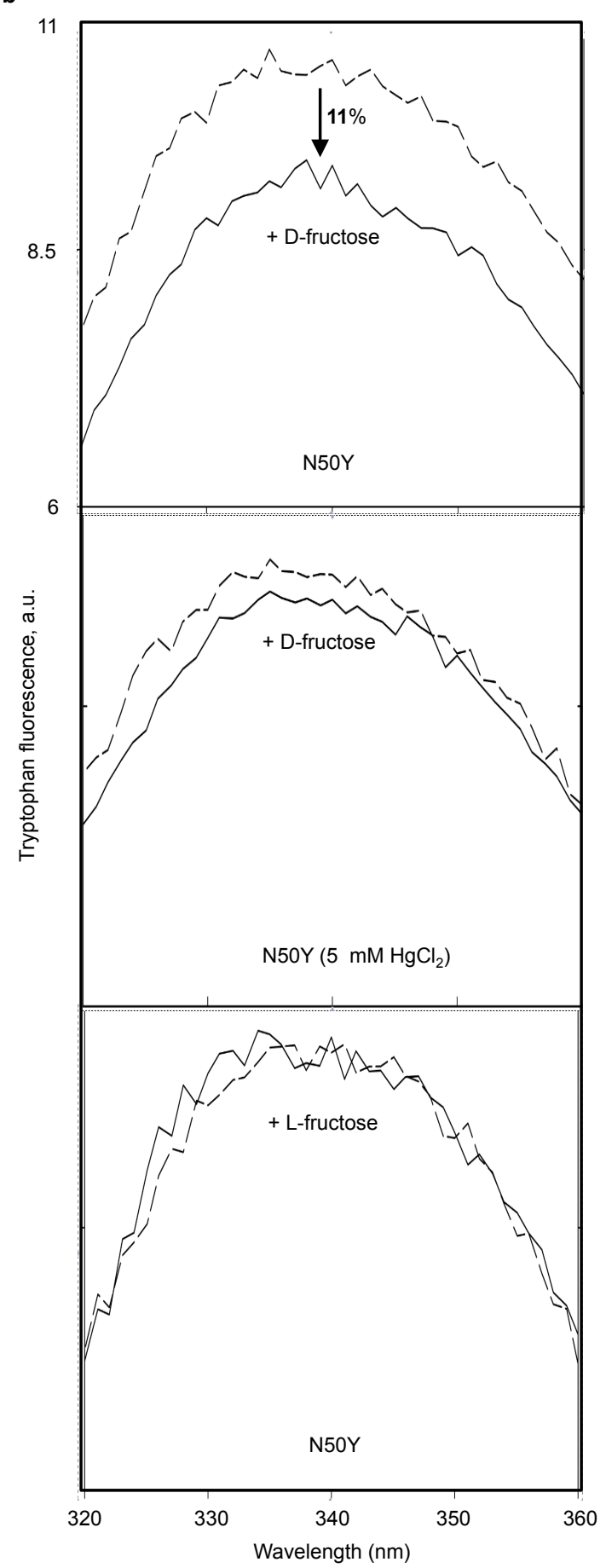

c

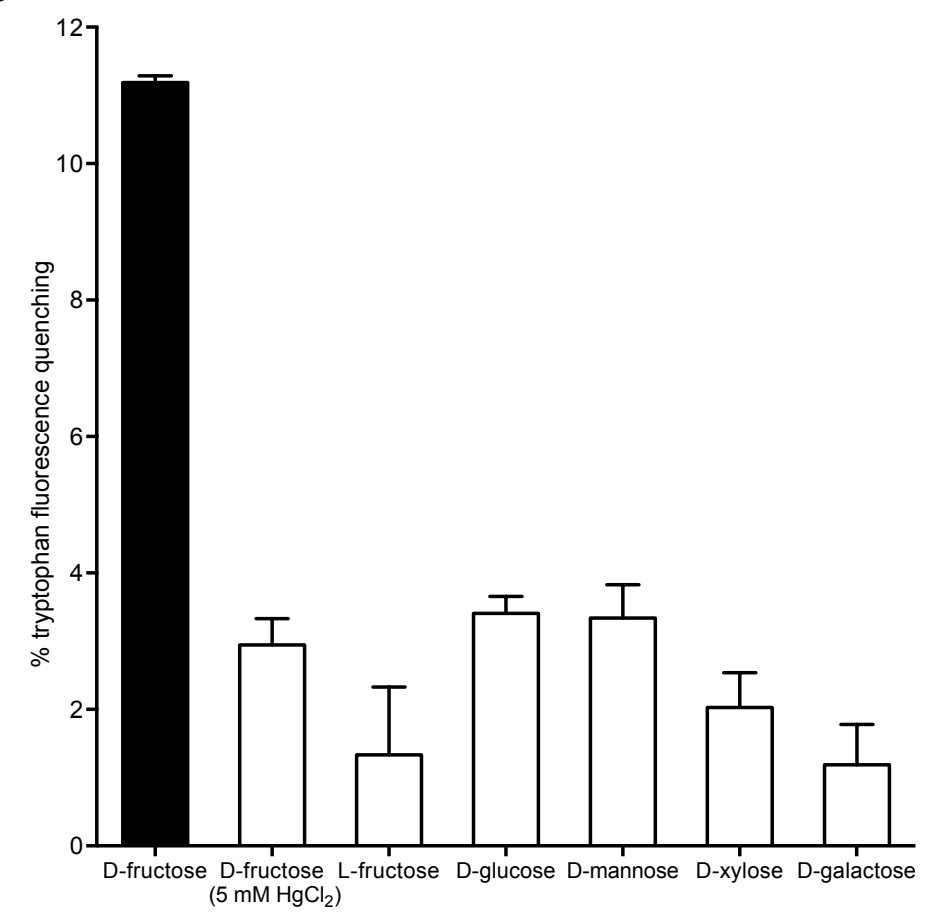

d

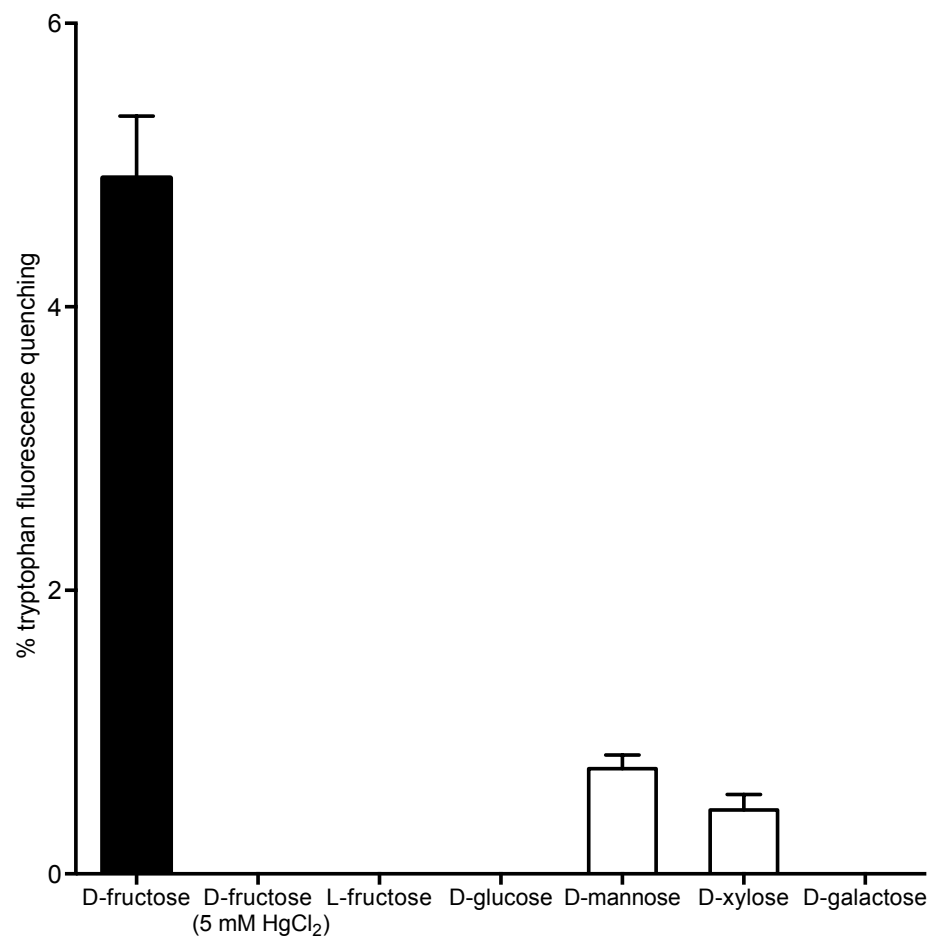


a

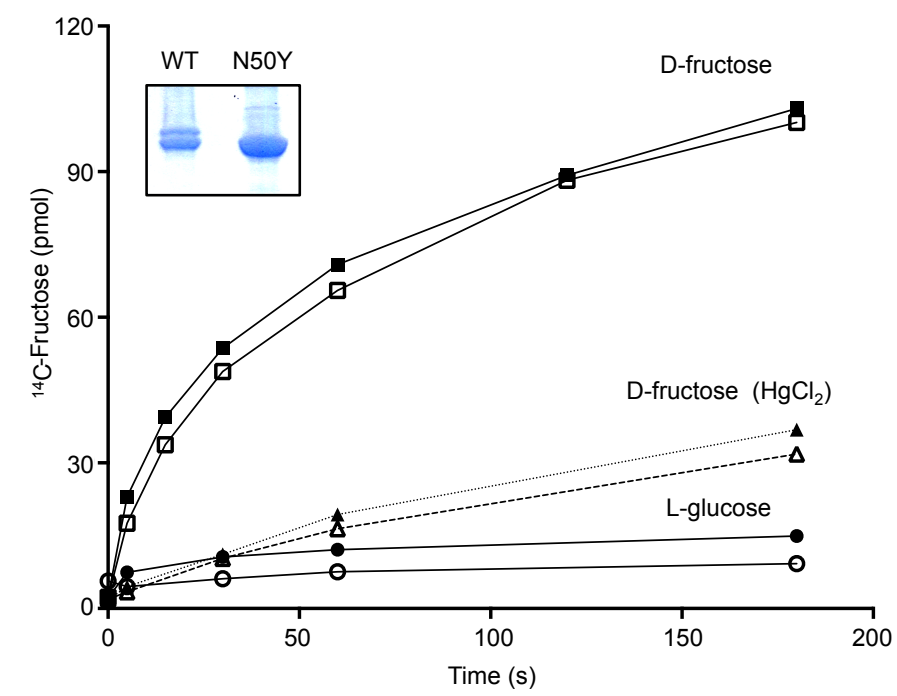

b

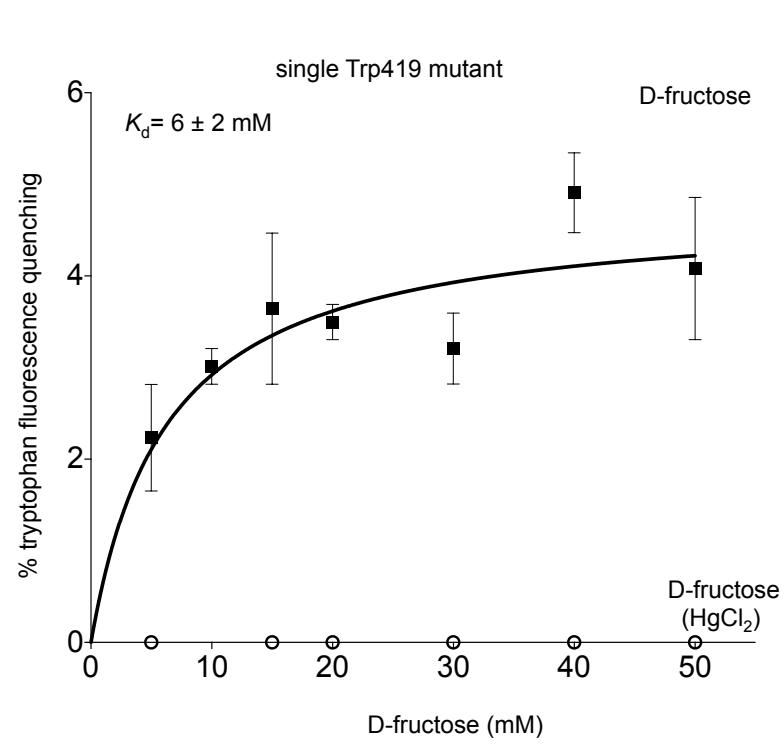

d

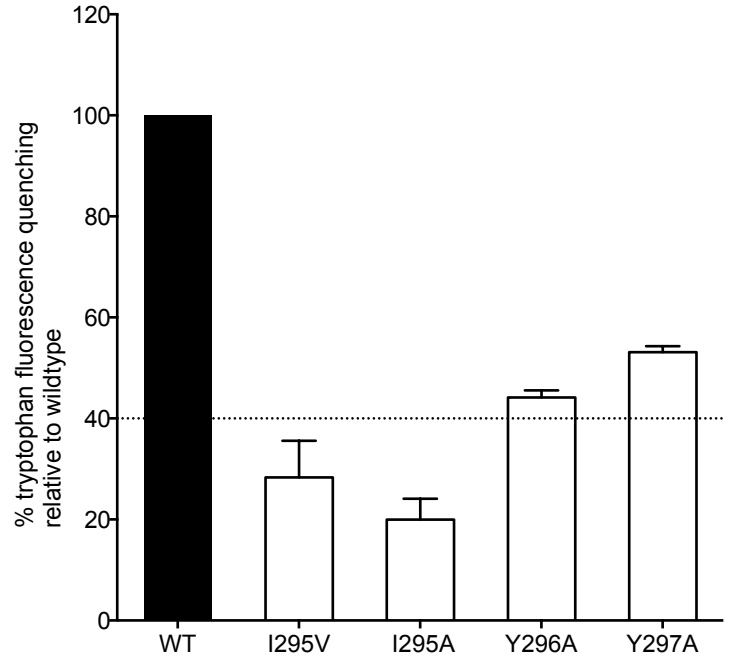

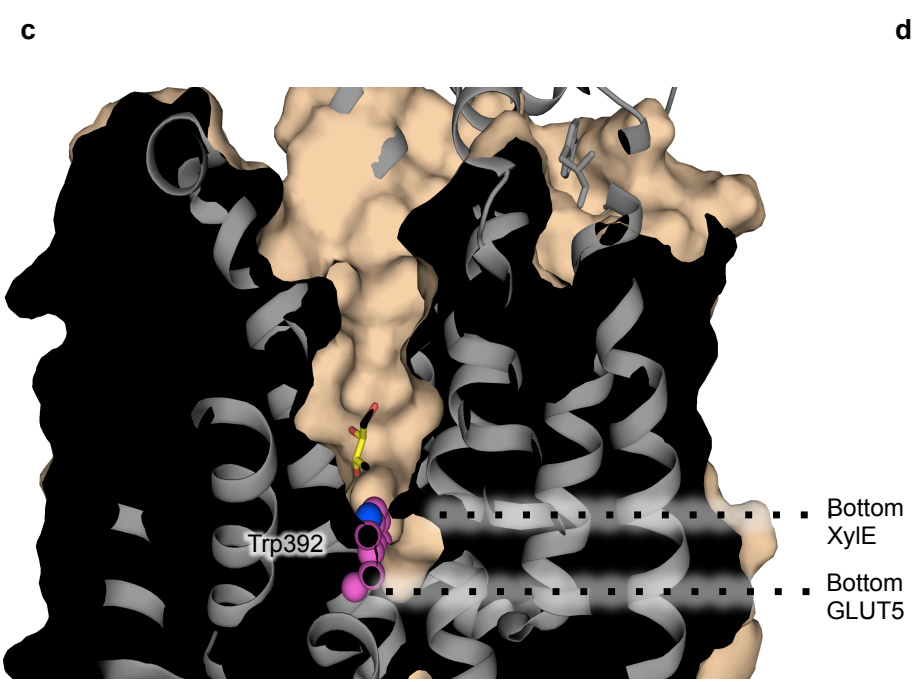

Extended Data Fig. 6 


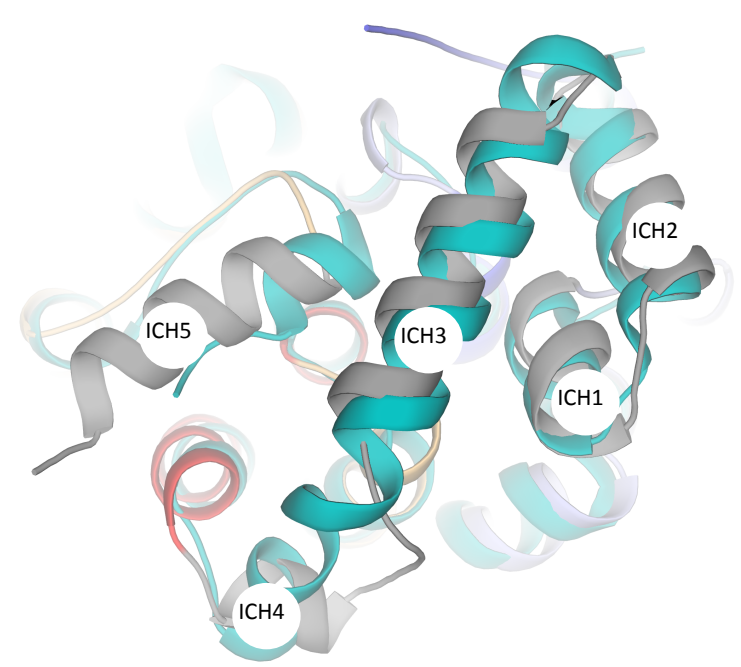

Extended Data Fig. 7

b

grey = outward-facing Glut5 teal $=$ outward-occluded XylE

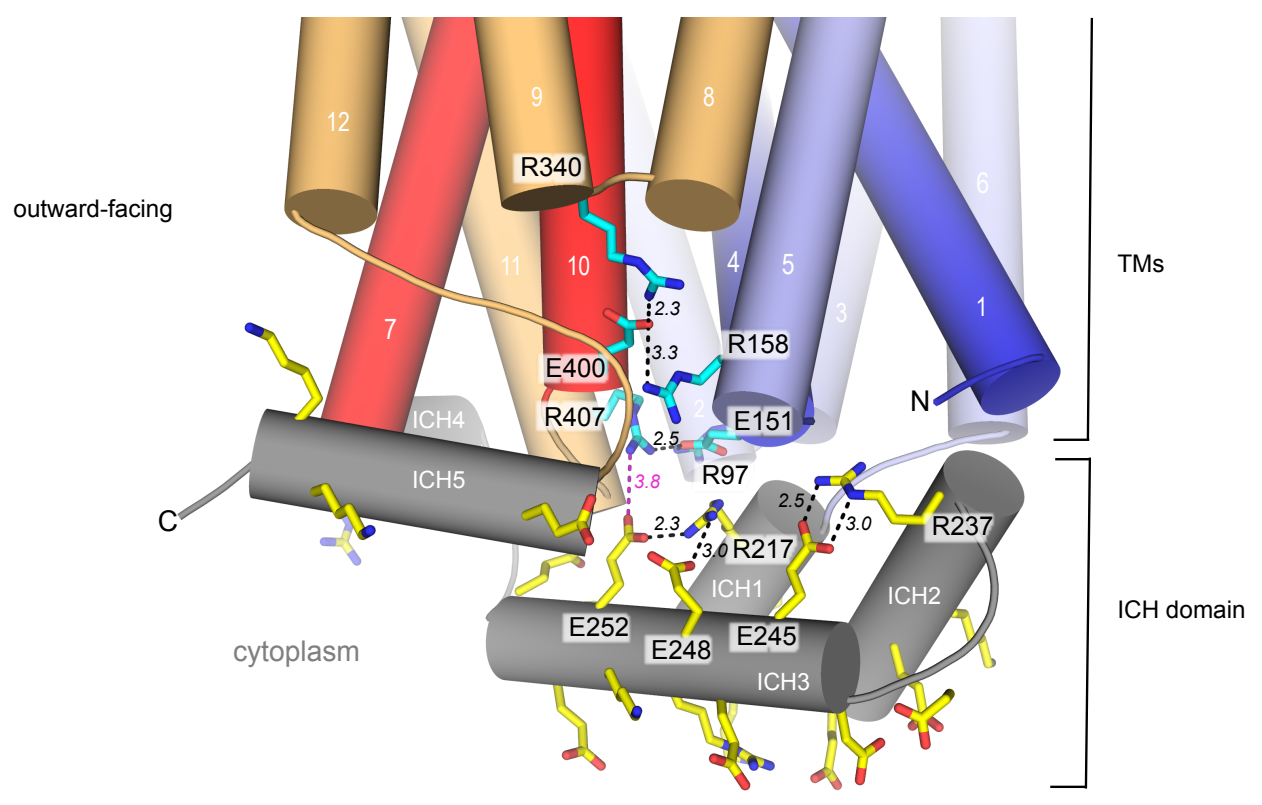

c

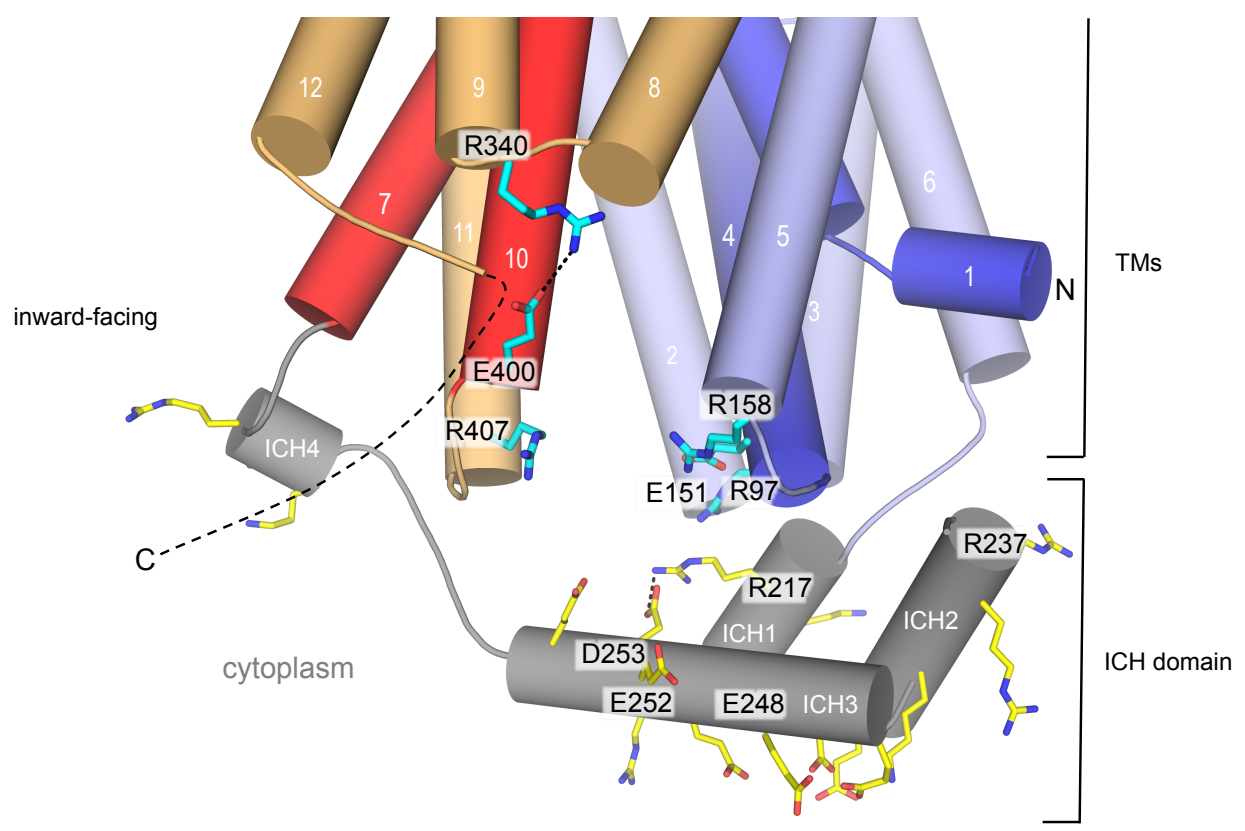


b

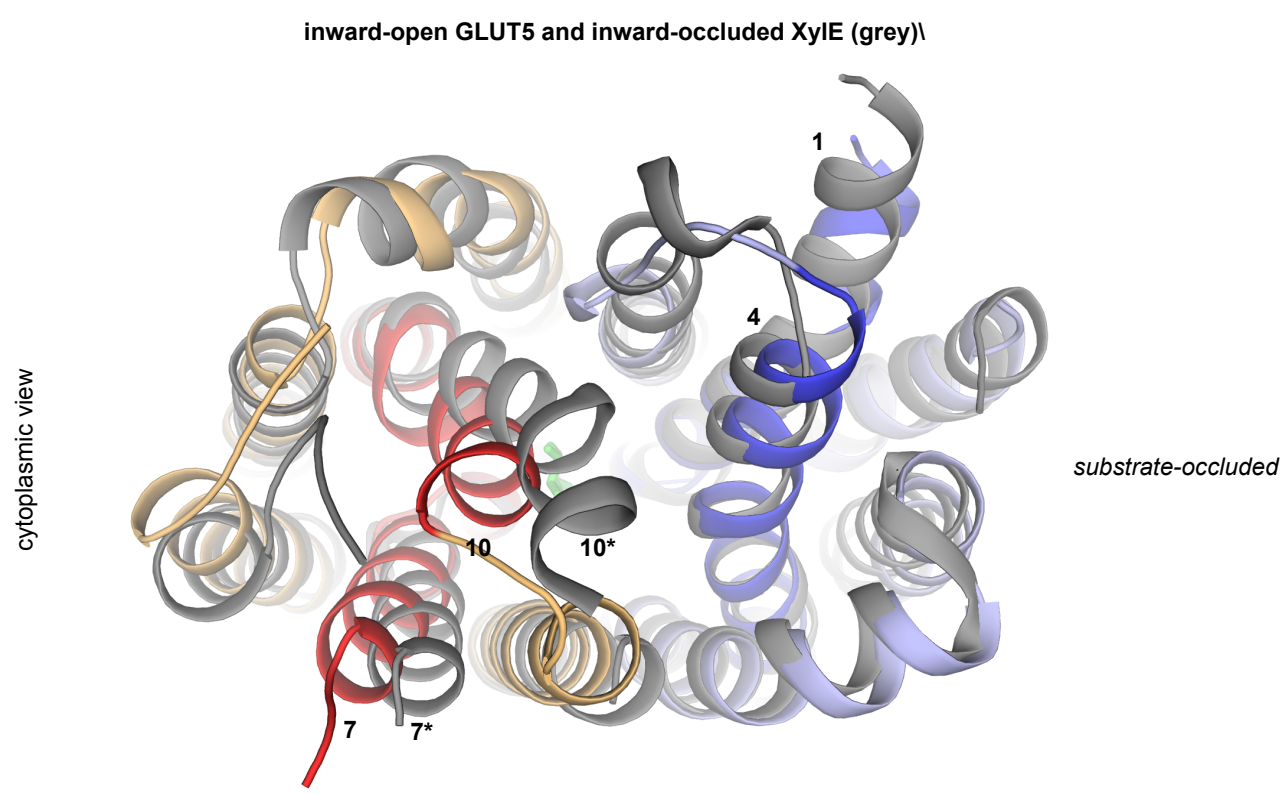

c

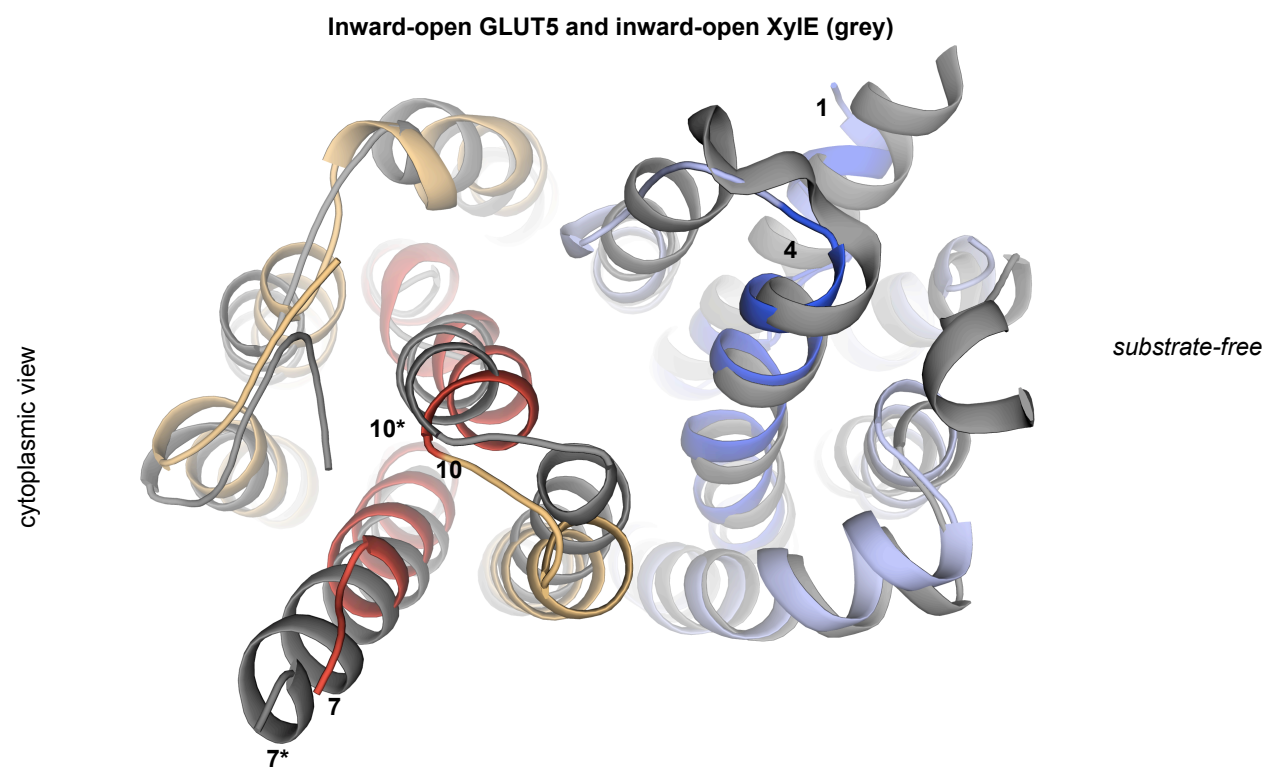

